IUCrJ

ISSN 2052-2525

BIOLOGY|MEDICINE

₹ Both authors contributed equally.

Keywords: collagen type IV; network assembly; (IV)NC1 hexamers; Goodpasture's disease; Alport's syndrome

\section{Structures of collagen IV globular domains: insight into associated pathologies, folding and network assembly. Corrigendum}

\author{
Patricia Casino, ${ }^{\mathrm{a}, \mathrm{b}, \mathrm{c} *} \neq$ Roberto Gozalbo-Rovira, ${ }^{\mathrm{d}, \mathrm{e}} \neq$ Jesús Rodríguez-Díaz, ${ }^{\mathrm{d}, \mathrm{e}}$ Surajit \\ Banerjee, ${ }^{\mathrm{f}}$ Ariel Boutaud, ${ }^{\mathrm{g}}$ Vicente Rubio, ${ }^{\mathrm{b}, \mathrm{c}}$ Billy G. Hudson, ${ }^{\mathrm{h}, \mathrm{i}, \mathrm{j}, \mathrm{k}, \mathrm{l}, \mathrm{m}, \mathrm{n}}$ Juan Saus, ${ }^{\circ}$ \\ Javier Cervera ${ }^{b, c, d}$ and Alberto Marina ${ }^{b, c *}$
}

${ }^{\mathrm{a}}$ Department of Biochemistry and Molecular Biology/ERI BIOTECMED, Universitat de València, Dr Moliner 50, Burjassot, 46100 Valencia, Spain, 'b Instituto de Biomedicina de Valencia, Consejo Superior de Investigaciones Científicas (IBV-CSIC), Jaume Roig 11, 46010 Valencia, Spain, ${ }^{\mathbf{c}}$ CIBER de Enfermedades Raras (CIBERER-ISCIII), Spain, dLaboratorio de Reconocimiento Molecular, Centro de Investigación Príncipe Felipe, Eduardo Primo Yúfera 3, 46012 Valencia, Spain, e Departamento de Microbiología, Facultad de Medicina at Universitat de València, Blasco Ibáñez 17, 46010 Valencia, Spain, 'Department of Defense, Center for Prostate Disease Research, Bethesda, Maryland, USA, 'BioStratum Inc., Durham, North Carolina, USA, hepartment of Medicine at Division of Nephrology and Hypertension, Vanderbilt University Medical Center, Nashville, TN 37232, USA, 'Center for Matrix Biology, Vanderbilt University Medical Center, Nashville, TN 37232, USA, 'Department of Biochemistry, Vanderbilt University Medical Center, Nashville, TN 37232, USA, kDepartment of Pathology, Microbiology and Immunology, Vanderbilt University Medical Center, Nashville, TN 37232, USA, 'Department of Cell and Developmental Biology, Vanderbilt University Medical Center, Nashville, TN 37232, USA, ' $\mathbf{m}$ Vanderbilt Ingram Cancer Center, Vanderbilt University Medical Center, Nashville, TN 37232, USA, ${ }^{\mathbf{n}}$ Vanderbilt Institute of Chemical Biology, Vanderbilt University Medical Center, Nashville, TN 37232, USA, and oDepartamento de Bioquímica y Biología Molecular at Facultad de Medicina y Odontología, Universitat de València, Blasco Ibáñez 15-17, 46010 Valencia, Spain. *Correspondence e-mail: patricia.casino@uv.es, amarina@ibv.csic.es

The article by Casino et al. [IUCrJ (2018). 5, 765-779] is corrected.

The following corrections to the article by Casino et al. (2018) are given.

(a) The name of one of the contributing authors, Surajit Banerjee, was incorrectly listed as Sreedatta Banerjee.

(b) The address of the author Surajit Banerjee, when the article was published, should have been Northeastern Collaborative Access Team and Department of Chemistry and Chemical Biology, Cornell University, Argonne, Illinois, USA.

\title{
References
}

Casino, P., Gozalbo-Rovira, R., Rodríguez-Díaz, J., Banerjee, S., Boutaud, A., Rubio, V., Hudson, B. G., Saus, J., Cervera, J. \& Marina, A. (2018). IUCrJ, 5, 765-779.

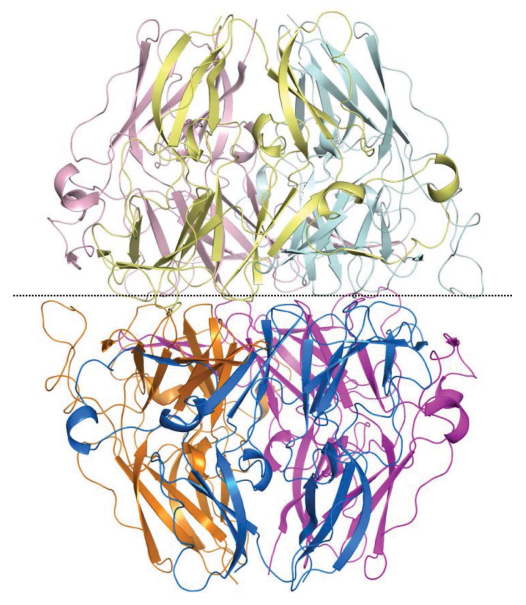

OPEN Ә ACCESS 
IUCrJ

ISSN 2052-2525

BIOLOGY|MEDICINE

Received 11 July 2018

Accepted 4 September 2018

Edited by J. Trewhella, University of Sydney, Australia

₹ Both authors contributed equally.

Keywords: collagen type IV; network assembly; (IV)NC1 hexamers; Goodpasture's disease; Alport's syndrome.

PDB references: collagen type IV $\alpha 1 \alpha 2 \alpha 1 \mathrm{NC} 1$, 5 nax; $\alpha 1 \mathrm{NC1}$, 5nay; $\alpha 2 \mathrm{NC} 1,5 \mathrm{nb} 2 ; \alpha 3 \mathrm{NC} 1$, $5 \mathrm{nb} 0 ; \alpha 4 \mathrm{NC} 1,5 \mathrm{nb} 1 ; \alpha 5 \mathrm{NC} 1,5 \mathrm{naz}$

Supporting information: this article has supporting information at www.iucrj.org

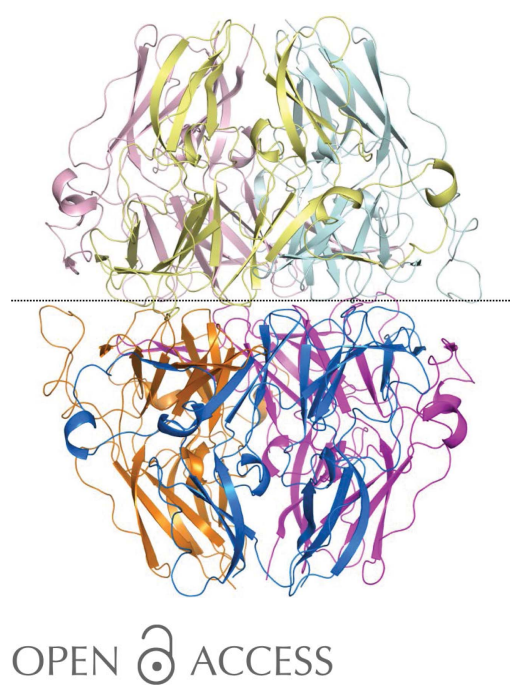

\section{Structures of collagen IV globular domains: insight into associated pathologies, folding and network assembly}

\author{
Patricia Casino, ${ }^{\mathrm{a}, \mathrm{b}, \mathrm{c} *}$ ‡ Roberto Gozalbo-Rovira, ${ }^{\mathrm{d}, \mathrm{e}} \neq$ Jesús Rodríguez-Díaz, ${ }^{\mathrm{d}, \mathrm{e}}$ \\ Sreedatta Banerjee, ${ }^{\mathrm{f}}$ Ariel Boutaud, ${ }^{\mathrm{g}}$ Vicente Rubio, ${ }^{\mathrm{b}, \mathrm{c}}$ Billy G. Hudson, ${ }^{\mathrm{h}, \mathrm{i}, \mathrm{j}, \mathrm{k}, \mathrm{l}, \mathrm{m}, \mathrm{n}}$ \\ Juan Saus, ${ }^{\circ}$ Javier Cervera ${ }^{b, c, d}$ and Alberto Marina ${ }^{b, c *}$
}

a Department of Biochemistry and Molecular Biology/ERI BIOTECMED, Universitat de València, Dr Moliner 50, Burjassot, 46100 Valencia, Spain, 'b Instituto de Biomedicina de Valencia, Consejo Superior de Investigaciones Científicas (IBV_CSIC), Jaume Roig 11, 46010 Valencia, Spain, ' CIBER de Enfermedades Raras (CIBERER-ISCIII), Spain, 'Laboratorio de Reconocimiento Molecular, Centro de Investigación Príncipe Felipe, Eduardo Primo Yúfera 3, 46012 Valencia, Spain, ${ }^{\mathbf{e}}$ Departamento de Microbiología, Facultad de Medicina at Universitat de València, Blasco Ibáñez 17, 46010 Valencia, Spain, 'Department of Defense, Center for Prostate Disease Research, Bethesda, Maryland, USA, 'BBioStratum Inc., Durham, North Carolina, USA, hepartment of Medicine at Division of Nephrology and Hypertension, Vanderbilt University Medical Center, Nashville, TN 37232, USA, 'Center for Matrix Biology, Vanderbilt University Medical Center, Nashville, TN 37232, USA, 'Department of Biochemistry, Vanderbilt University Medical Center, Nashville, TN 37232, USA, ${ }^{\mathbf{k}}$ Department of Pathology, Microbiology and Immunology, Vanderbilt University Medical Center, Nashville, TN 37232, USA, 'Department of Cell and Developmental Biology, Vanderbilt University Medical Center, Nashville, TN 37232, USA, ${ }^{\mathbf{m}}$ Vanderbilt Ingram Cancer Center, Vanderbilt University Medical Center, Nashville, TN 37232, USA, ${ }^{\mathbf{n}}$ Vanderbilt Institute of Chemical Biology, Vanderbilt University Medical Center, Nashville, TN 37232, USA, and o Departamento de Bioquímica y Biología Molecular at Facultad de Medicina y Odontología, Universitat de València, Blasco Ibáñez 15-17, 46010 Valencia, Spain. *Correspondence e-mail: patricia.casino@uv.es, amarina@ibv.csic.es

Basement membranes are extracellular structures of epithelia and endothelia that have collagen IV scaffolds of triple $\alpha$-chain helical protomers that associate end-to-end, forming networks. The molecular mechanisms by which the noncollagenous C-terminal domains of $\alpha$-chains direct the selection and assembly of the $\alpha 1 \alpha 2 \alpha 1$ and $\alpha 3 \alpha 4 \alpha 5$ hetero-oligomers found in vivo remain obscure. Autoantibodies against the noncollagenous domains of the $\alpha 3 \alpha 4 \alpha 5$ hexamer or mutations therein cause Goodpasture's or Alport's syndromes, respectively. To gain further insight into oligomer-assembly mechanisms as well as into Goodpasture's and Alport's syndromes, crystal structures of noncollagenous domains produced by recombinant methods were determined. The spontaneous formation of canonical homohexamers (dimers of trimers) of these domains of the $\alpha 1, \alpha 3$ and $\alpha 5$ chains was shown and the components of the Goodpasture's disease epitopes were viewed. Crystal structures of the $\alpha 2$ and $\alpha 4$ noncollagenous domains generated by recombinant methods were also determined. These domains spontaneously form homo-oligomers that deviate from the canonical architectures since they have a higher number of subunits (dimers of tetramers and of hexamers, respectively). Six flexible structural motifs largely explain the architectural variations. These findings provide insight into noncollagenous domain folding, while supporting the in vivo operation of extrinsic mechanisms for restricting the self-assembly of noncollagenous domains. Intriguingly, Alport's syndrome missense mutations concentrate within the core that nucleates the folding of the noncollagenous domain, suggesting that this syndrome, when owing to missense changes, is a folding disorder that is potentially amenable to pharmacochaperone therapy.

\section{Introduction}

The extracellular microenvironment plays a pivotal role in tissue genesis, architecture and function. A defining morphological feature of these microenvironments is the basement membrane $(\mathrm{BM})$, an ancient and specialized form of extracellular matrix that is conserved from cnidarians to humans. 
BMs underlie epithelia and endothelia (Hagios et al., 1998; Rhodes \& Simons, 2007; Fidler et al., 2017) and ensheath muscle, fat and Schwann cells (Campbell \& Stull, 2003; Sanes, 2003; Sillat et al., 2012; Court et al., 2006). BMs function as supramolecular scaffolds that compartmentalize and provide structural integrity to tissues, guide cell migration and adhesion, delineate apical-basal polarity and modulate cell differentiation during development (Hynes, 2009; Pastor-Pareja \& Xu, 2011; Daley \& Yamada, 2013).

Type IV collagen is a major constituent of BMs. This evolutionarily conserved protein forms networks and plays a crucial structural role in the maintenance of BM architecture (Brown et al., 2017; Fidler et al., 2018). In addition, it serves as a ligand for cell-surface integrins, thus influencing cell adhesion, migration and differentiation (Wang et al., 2008). Not surprisingly, mutations in collagen IV cause BM destabilization and tissue dysfunction, and are associated with inborn defects such as Alport's syndrome and cerebral haemorrhage (Hudson et al., 2003; Kuo et al., 2012; Gould et al., 2005). Furthermore, Goodpasture's disease results from a selective antibody-mediated aggression to collagen IV, specifically to epitopes localized in the globular noncollagenous domains (NC1s) of collagen IV $\alpha$-chains 3 and 5 (Hudson et al., 2003; Cui et al., 2016). These are two of the six types of collagen IV chains that exist in humans [designated $\alpha 1-\alpha 6(\mathrm{IV}) \mathrm{NC} 1 \mathrm{~s}$; in the following the (IV) will be omitted for simplicity]. The Goodpasture epitopes remain to be structurally characterized, but they are considered to be cryptic, becoming immunovisible as a consequence of solvent exposure or viral infection (Pedchenko et al., 2010). These collagen IV-related disorders can be deadly as a result of impaired BM function.

Unveiling how collagen IV is organized in BMs seems to be crucial in order to understand the molecular architectures of BMs and to clarify the pathogenesis of BM-related diseases. As previously mentioned, six different types of homologous polypeptide chains of similar lengths (1669-1712 residues) can be found in collagen type IV. These chains $(\alpha 1-\alpha 6)$, encoded by paralogous genes, are composed of a short N-terminal domain termed the $7 \mathrm{~S}$ domain ( $\sim 25$ amino acids), a long central collagenous domain ( $\sim 1400$ amino acids) and a noncollagenous globular C-terminal domain of around 230 amino acids [abbreviated as (IV)NC1; the (IV) will be omitted in the following; Timpl et al., 1981; Borza et al., 2001]. Three $\alpha$-chains associate into a protomer with a long parallel triple-helical shaft and nonhelical ends. In the collagen IV network found in $\mathrm{BMs}$, different protomers interconnect by end-to-end interactions. At the C-termini, interactions occur between the trimeric $\mathrm{NC1}$ domains of two protomers, forming a hexamer of these domains reinforced by sulfilimine cross-links (Vanacore et al., 2009). At the $\mathrm{N}$-termini, four protomers interact through their $7 \mathrm{~S}$ domains, forming a $7 \mathrm{~S}$ dodecamer reinforced by aldehyde-derived cross-links (Anazco et al., 2016). In this way, a combination of $\mathrm{C}$-terminal hexameric and $\mathrm{N}$-terminal dodecameric interactions generates the three-dimensional collagen IV scaffold to which other components of the BMs bind (Hudson et al., 2003; Brown et al., 2017). Intriguingly, only three distinct protomers, $\alpha 1 \alpha 2 \alpha 1, \alpha 3 \alpha 4 \alpha 5$ and $\alpha 5 \alpha 6 \alpha 5$ (abbreviated in the following as $\alpha 121, \alpha 345$ and $\alpha 565$, respectively), forming three distinct networks, $\alpha 121, \alpha 345$ and $\alpha 121-\alpha 565$, are known to occur in BMs (Boutaud et al., 2000; Borza et al., 2001; Gunwar et al., 1998). Previously unrecognized collagen IV $\alpha 125$ chain combinations have recently been found in various cancer cell lines, which form networks of protomers of $\alpha 1 \alpha 2$ chains and $\alpha 5$-chain homoprotomers (Revert et al., 2018). The formation of these novel collagen IV networks is dependent on an exportable protein kinase, the Goodpasture antigen-binding protein (Raya et al., 1999; Revert et al., 2008, 2018), pointing to the involvement of the cellular machinery in regulating the organization of collagen IV networks.

The assembly of the collagen IV network involves two distinct stages of $\alpha$-chain oligomerization. Three $\alpha$-chains first associate intracellularly, forming protomers, and the protomers then associate end-to-end outside the cell, generating extracellular networks. Hints on the assembly mechanisms were inferred (i) from the crystal structure of the $\alpha 121 \mathrm{NC} 1$ hexamer isolated from natural sources (Sundaramoorthy et al., 2002; Than et al., 2002; Vanacore et al., 2004), (ii) from the ability of recombinant $\alpha 1 \mathrm{NC} 1$ chains to selectively assemble into hexamers (Boutaud et al., 2000) and (iii) from refolding experiments with NC1 hexamers (Dölz et al., 1988). Recent studies in one of our laboratories (Cummings et al., 2016) revealed that the $\mathrm{NC1}$ domains function as recognition modules, directing the selection and assembly of $\alpha$-chains into protomers and networks. In turn, chloride ions activate a molecular switch present in $\mathrm{NC1}$ domains that triggers the oligomerization of protomers into networks. However, how $\mathrm{NC} 1$ recognition modules direct $\alpha$-chain oligomerization has so far been ignored.

To obtain further insight into collagen IV network assembly and to advance the characterization of the molecular bases of Goodpasture's and Alport's syndromes, we generated various $\mathrm{NC1}$ domains via recombinant technology and used protein crystallography to identify and structurally characterize the oligomers produced by these domains. Using this approach, while attempting to produce crystals of the $\alpha 345 \mathrm{NC} 1$ heterohexamer that predominates in the BM of the kidney, we solved the three-dimensional structure of the recently reported homohexamer of $\alpha 5 \mathrm{NC1}$ domains (Revert et al., 2018). In addition, we crystallized and determined the structures of the homohexamers spontaneously formed by recombinantly produced $\alpha 1 \mathrm{NC1}$ and $\alpha 3 \mathrm{NC} 1$ domains. Interestingly, when carrying out similar studies with crystals of the $\alpha 2 \mathrm{NC1}$ and $\alpha 4 \mathrm{NC} 1$ domains, oligomers were spontaneously formed that were architecturally similar to the hexamers observed for the other chains except that they were dimers of tetramers (octamers) or of hexamers, respectively, suggesting the existence of restrictions that limit the oligomers produced in vivo. We also validated the use of self-assembled recombinantly produced $\mathrm{NC1}$ domains for structural studies by reproducing in vitro the $\alpha 121$ heterohexamer previously obtained from natural sources (Sundaramoorthy et al., 2002; Than et al., 2002; Vanacore et al., 2004). The information gathered provided insights into the structural features involved in the folding, 
selection and oligomerization of collagen IV chains to form a protomer. Furthermore, we provide the first snapshots of the components of the Goodpasture autoantigen. We also provide insight into Alport's syndrome, helping to rationalize the structural bases of the effects of the mutations reported in this disease that map to $\mathrm{NC} 1$ domains, and concluding that of these mutations, those that are amino-acid substitutions may cause disease by inducing misfolding, thus opening the way to attempting therapy using pharmacochaperones.

\section{Methods}

\subsection{Cloning, protein expression and purification}

We obtained a cDNA library from HEK293 cells (a commercial cell line derived from human embryonic kidney) by retrotranscription with SuperScript reverse transcriptase (Invitrogen) of mRNA extracted from these cells using TRIzol (Invitrogen). This cDNA library was used as template for PCR amplification of the $\alpha 1 \mathrm{NC} 1, \alpha 2 \mathrm{NC} 1, \alpha 4 \mathrm{NC} 1$ and $\alpha 5 \mathrm{NC} 1$ domains using a high-fidelity thermophilic DNA polymerase ( $P f u$ polymerase, Stratagene) and the primers listed in Supplementary Table S1. The PCR-amplified coding sequences for these $\mathrm{NC} 1$ domains were used in a second round of PCR amplification with additional primers (Supplementary Table S1) to introduce an $\mathrm{N}$-terminal BM40 secretion peptide followed by a FLAG tag preceding the sequences of the indicated NC1 domains (the residues forming each of these domains are indicated in Supplementary Table S2). These BM40-FLAG-tagged domains were then subcloned into pFastBac1 using BamHI and SacI sites for the cloning of $\alpha 2 \mathrm{NC} 1$ and $\alpha 5 \mathrm{NC} 1$, and $\mathrm{XhoI}$ and $\mathrm{KpnI}$ sites for the cloning of $\alpha 1 \mathrm{NC} 1$ and $\alpha 4 \mathrm{NC} 1$. In the case of $\alpha 3 \mathrm{NC} 1$, the BM40-FLAGtagged $\alpha 3 \mathrm{NC} 1$ coding sequence was extracted by SacI digestion from the previously reported F $\alpha 3$ ANU-pRC-CMV vector (Gozalbo-Rovira et al., 2013; Netzer et al., 1999) and was then subcloned into the pFastBac1 vector.

The resulting pFastBac1 vectors with the indicated inserts were transformed into DH10Bac competent cells and the recombinant baculoviruses were obtained following the instructions provided by the supplier of the baculovirus expression system (Bac-to-Bac Baculovirus Expression System, Invitrogen). The recombinant baculoviruses were used to produce all of the $\mathrm{NC} 1$ domains in $\mathrm{Sf} 9$ insect cells, as previously described for $\alpha 2 \mathrm{NC1}$ and $\alpha 3 \mathrm{NC} 1$ (Gozalbo-Rovira et al., 2013). All purification steps were carried out at $4^{\circ} \mathrm{C}$. Supernatants from 11 cultures underwent ultracentrifugation $(1 \mathrm{~h}, 160000 \mathrm{~g})$ and the equivalent of $2 \mathrm{ml}$ column-packed ANTI-FLAG M2 affinity gel (Sigma-Aldrich/Merck) was added. After packing the gel into a column, it was washed with $50 \mathrm{ml} 50 \mathrm{~m} M$ Tris- $\mathrm{HCl} \mathrm{pH}$ 7.4, $0.15 \mathrm{M} \mathrm{NaCl}$. The NC1s were eluted with $10 \mathrm{ml}$ of the same solution supplemented with $0.1 \mathrm{mg} \mathrm{ml}^{-1}$ soluble FLAG peptide (DYKDDDDK). The eluted proteins were concentrated and the FLAG peptide was removed by repeated runs of concentration and dilution in elution buffer without peptide using Amicon Ultra-4 10K Centrifugal Filter Devices (Merck-Millipore). Around $1 \mathrm{mg}$ of soluble recombinant protein was usually obtained per litre of culture.

In addition to being produced in the baculovirus/insect-cell expression system, $\alpha 2 \mathrm{NC1}$ was also obtained in one of our laboratories as an N-terminally FLAG-tagged fusion protein using HEK293 cells for expression and purification, as described elsewhere (Sado et al., 1998).

\subsection{Protein crystallization and structure determination}

Crystals of $\alpha 2 \mathrm{NC1}$ (expressed in HEK293 cells) and of $\alpha 1 \mathrm{NC} 1, \alpha 3 \mathrm{NC} 1, \alpha 4 \mathrm{NC} 1, \alpha 5 \mathrm{NC} 1$ and $\alpha 121 \mathrm{NC} 1$ (expressed in insect cells) were obtained by the sitting-drop vapourdiffusion technique at $21^{\circ} \mathrm{C}$ using $0.4 \mu \mathrm{l}$ protein solution (7.4 $\mathrm{mg} \mathrm{ml}^{-1}$ protein in $50 \mathrm{mM}$ Tris- $\mathrm{HCl} \mathrm{pH} \mathrm{7.4,} 0.15 \mathrm{M} \mathrm{NaCl}$, except for $\alpha 3 \mathrm{NC1}$, for which the $\mathrm{NaCl}$ concentration was $0.5 \mathrm{M}$ ) mixed with $0.4 \mu \mathrm{l}$ of the crystallization solution indicated in Table 1 . For the $\alpha 121 \mathrm{NC} 1$ crystals a 2:1 mixture (in terms of mass) of $\alpha 1 \mathrm{NC} 1$ and $\alpha 2 \mathrm{NC} 1$ chains was used, whereas for the $\alpha 5 \mathrm{NC} 1$ crystals a 1:1:1 mixture of $\alpha 3 \mathrm{NC} 1, \alpha 4 \mathrm{NC} 1$ and $\alpha 5 \mathrm{NC} 1$ chains was used. The crystal-harvesting solutions used for cryopreservation are listed in Table 1. X-ray diffraction was carried out at $100 \mathrm{~K}$ using the indicated beamlines and wavelengths (Table 1) at the European Synchrotron Facility (ESRF), Grenoble, France or the ALBA synchrotron, Cerdanyola, Barcelona, Spain. Crystallographic data sets were processed and scaled using either MOSFLM and SCALA (CCP4 suite; Winn et al., 2011) or the XDS program package (Kabsch, 2010). Data-collection details and unit-cell parameters are given in Table 1. Phases were obtained for all of the crystals by molecular replacement with Phaser (McCoy et al., 2007) using the structure of one $\alpha 1 \mathrm{NC} 1$ domain of the bovine $\alpha 121$ crystal structure as a search model (PDB entry 1t60; Vanacore et al., 2004). For the $\alpha 3 \mathrm{NC} 1$ and $\alpha 121 \mathrm{NC} 1$ crystals, initial data processing with SCALA indicated $39-40 \%$ twinning in both cases. By alternating cycles of refinement with REFMAC5 (Murshudov et al., 2011) with manual model building using Coot (Emsley et al., 2010) and, in the case of the $\alpha 3 \mathrm{NC} 1$ and $\alpha 121 \mathrm{NC} 1$ crystals by applying twinning refinement as implemented in REFMAC5, final models at 1.8-2.8 resolution, depending on the crystal, were obtained (see Table 1). The crystals exhibited good quality-control parameters and excellent stereochemistry (the Ramachandran plot distributions of favoured/allowed/disallowed residues for $\alpha 1 \mathrm{NC} 1, \alpha 2 \mathrm{NC} 1, \alpha 3 \mathrm{NC} 1, \alpha 4 \mathrm{NC} 1, \alpha 5 \mathrm{NC} 1$ and $\alpha 121 \mathrm{NC} 1$ were $97.7 / 2.3 / 0,97.9 / 2.1 / 0,97.7 / 2.3 / 0,95.3 / 4.5 / 0.2,97.8 / 2.2 / 0$ and $96.3 / 3.7 / 0 \%$, respectively). The structures and structure factors have been deposited in the PDB as entries 5nay, 5nb2, 5nb0, $5 \mathrm{nb} 1,5 \mathrm{naz}$ and 5 nax for the $\alpha 1 \mathrm{NC} 1, \alpha 2 \mathrm{NC} 1, \alpha 3 \mathrm{NC} 1, \alpha 4 \mathrm{NC} 1$, $\alpha 5 \mathrm{NC} 1$ and $\alpha 121 \mathrm{NC} 1$ crystals, respectively.

\subsection{Residue numbering for NC1 domains}

We have followed the convention used for the previously reported structures of $\alpha 121 \mathrm{NC} 1$ heterohexamers obtained from BMs (PDB entries 1li1, 1t60 and 1t61; Than et al., 2002; Vanacore et al., 2004). Correspondences with the residue 
Table 1

Crystallographic data and refinement statistics for crystals of recombinant $\mathrm{NC} 1$ chains and their mixtures.

Values in parentheses are for the highest resolution shell.

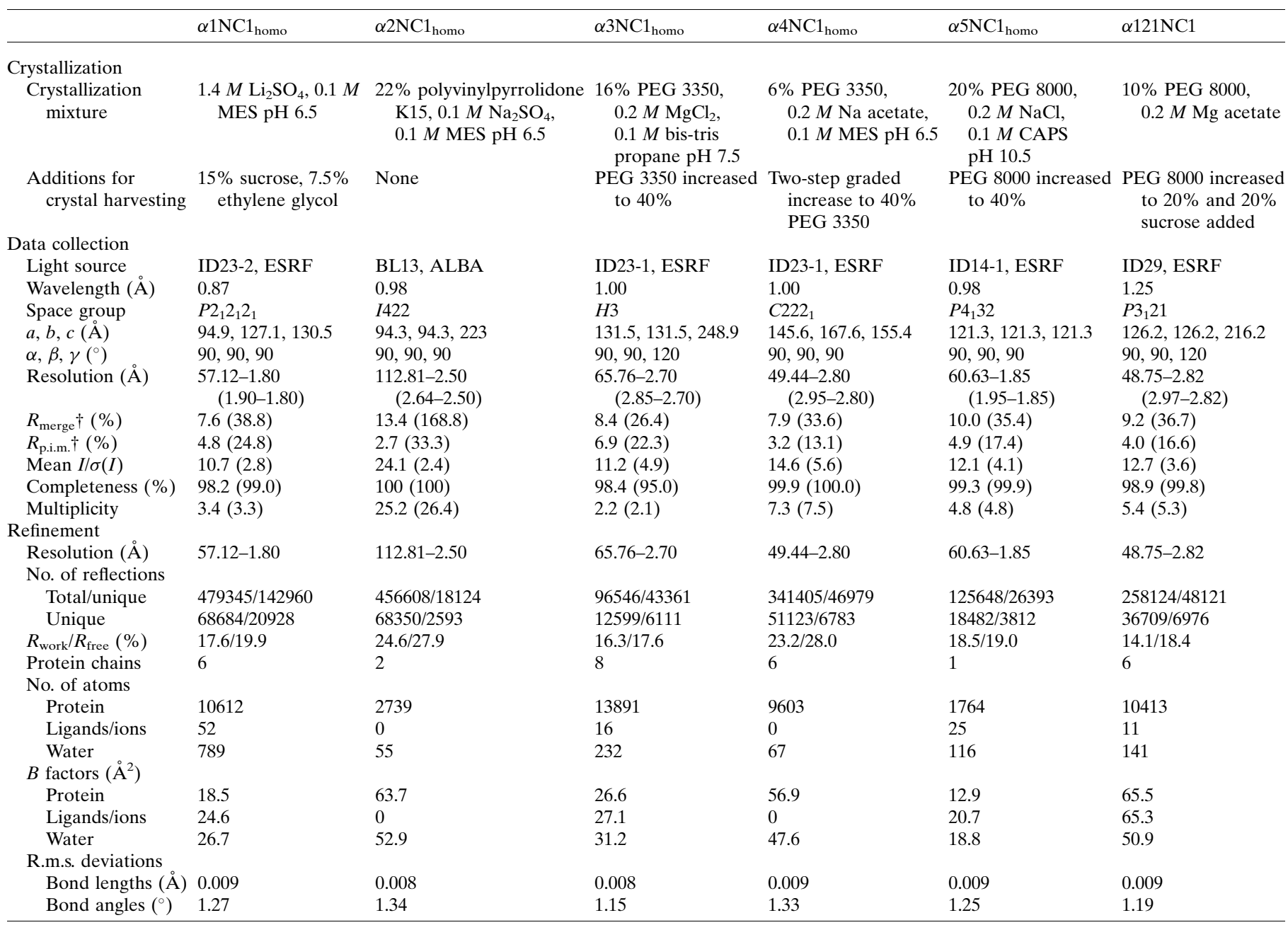

$\dagger$ A single crystal was used for each structure.

numbering for each complete $\alpha$-chain are provided in Supplementary Table S2.

\subsection{Other methods}

Structure-based alignments, structural superimpositions and analysis of protein contacts were performed with MUSTANG (Konagurthu et al., 2006; Papadopoulos \& Agarwala, 2007), SUPERPOSE and NCONT (CCP4 suite, Winn et al., 2011), respectively. Figures were produced using PyMOL (http://www.pymol.org).

Size-exclusion chromatography was performed at $20^{\circ} \mathrm{C}$ using a Superdex S200 (10/300) column fitted to an ÅKTA 900 FPLC system (column and instrument from GE Healthcare, Barcelona, Spain) using $50 \mathrm{~m} M$ Tris- $\mathrm{HCl}$ pH 7.4, 0.15 $M \mathrm{NaCl}$

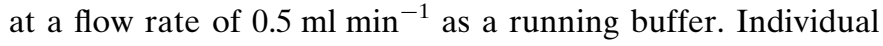
$\mathrm{NC} 1$ chains were incubated overnight at $20^{\circ} \mathrm{C}$ to allow oligomerization before injection, continuously monitoring the absorbance at $280 \mathrm{~nm}$ in the effluent.
Surface plasmon resonance (SPR) assays were performed using a Biacore T100 instrument (GE Healthcare). $\alpha 3 \mathrm{NC} 1$ or $\alpha 5 \mathrm{NC1}$ was immobilized on CM5 sensor chips (GE Healthcare) using amine-coupling chemistry by passing $1 \mu \mathrm{g} \mathrm{ml}^{-1}$ solutions of either $\mathrm{NC1}$ chain in $10 \mathrm{~m} M$ sodium acetate $\mathrm{pH} 3$ to attain a level of attachment of $\sim 1000$ resonance units (RU). The assays were performed at $25^{\circ} \mathrm{C}$ by passing a solution consisting of $10 \mathrm{~m} M$ Tris- $\mathrm{HCl} \mathrm{pH} 7.4,0.15 M \mathrm{NaCl}, 3 \mathrm{~m} M$ ethylenediaminetetraacetic acid, $0.005 \%$ surfactant P20 (GE Healthcare) including the indicated concentrations of $\alpha 3 \mathrm{NC} 1$ or $\alpha 5 \mathrm{NC1}$ at a flow rate of $10 \mu \mathrm{min}^{-1}$ for $300 \mathrm{~s}$, followed by a $300 \mathrm{~s}$ dissociation time with protein-free fluid. Between runs, the chip was regenerated by washing with $20 \mathrm{~m} M \mathrm{NaOH}$ at a flow rate of $75 \mu \mathrm{min}^{-1}$ for $20 \mathrm{~s}$ followed by re-equilibration in running buffer. BIAevaluation 2.0.3 (GE Healthcare) was used to extract the kinetic data and to estimate $K_{\mathrm{d}}$ values.

SDS-PAGE was performed according to Laemmli (1970). Coomassie Blue (Bradford, 1976) was used for protein determination using a commercial reagent (Bio-Rad, California, USA) with bovine serum albumin as a standard. 


\section{Results}

3.1. In vitro self-assembly of recombinantly produced chains generates the $\alpha 5 \mathrm{NC} 1$ homohexamer

In an attempt to visualize the Goodpasture antigens, we tried to crystallize the $\alpha 345 \mathrm{NC} 1$ heterohexamer by using an equimolecular mixture of pure recombinant $\alpha 3 \mathrm{NC} 1, \alpha 4 \mathrm{NC} 1$ and $\alpha 5 \mathrm{NC} 1$ domains (Supplementary Fig. S1). We obtained crystals that diffracted X-rays to $1.85 \AA$ resolution but that only revealed homohexamers of $\alpha 5 \mathrm{NC} 1$ chains (Table 1 and Fig. 1a), most likely representing the novel $\alpha 5 \mathrm{NC} 1$ homohexamer recently reported in cancer cell lines (Revert et al., 2018). Size-exclusion chromatography experiments confirmed a strong tendency of $\alpha 5 \mathrm{NC} 1$ to hexamerize: $\sim 80 \%$ of this domain was self-organized as hexamers in solution, while isolated $\alpha 3 \mathrm{NC} 1$ or $\alpha 4 \mathrm{NC} 1$ eluted as monomers (Supplementary Fig. S2a).

The architecture of the $\alpha 5 \mathrm{NC} 1$ homohexamer (hereafter referred to as $\alpha 5 \mathrm{NC}_{\text {homo }}$ ) is essentially the same as that of the $\alpha 121 \mathrm{NC} 1$ hexamer obtained from natural sources (Sundaramoorthy et al., 2002; Than et al., 2002; Vanacore et al., 2009). $\alpha 5 \mathrm{NC1}_{\text {homo }}$ was shaped as a prolate ellipsoid of $\sim 92 \times \sim 63 \AA$ (Figs. $1 a$ and 2, Supplementary Fig. S3a and Table S3). It was composed of two trimeric protomers meeting front-to-front at the equatorial plane of the ellipsoid in a nearly planar interaction surface. The subunit fold (Fig. 1b) showed a tandem arrangement of two similarly folded subdomains (hereafter referred to as $\mathrm{N}$-sub and $\mathrm{C}$-sub), as expected for the collagen IV NC1-domain fold (Pfam PF01413; https://pfam.xfam.org/ family/PF01413; Supplementary Table S4). Thus, each subdomain of the $\alpha 5 \mathrm{NC} 1$ subunit contains one $\alpha$-helix and ten $\beta$-strands forming two antiparallel $\beta$-sheets (sheets I and II in $\mathrm{N}$-sub and $\mathrm{I}^{\prime}$ and $\mathrm{II}^{\prime}$ in $\mathrm{C}$-sub; a prime identifies the elements of $\mathrm{C}$-sub; Fig. $1 b$ ). In each protomer, the I and $\mathrm{I}^{\prime}$ sheets (the topologies of these sheets are $\beta 1 \beta 10 \beta 2 \beta 5$ and $\beta 1^{\prime} \beta 10^{\prime} \beta 2^{\prime} \beta 5^{\prime}$, respectively) of the three subunits alternate, forming the polar layers of the prolate ellipsoid (the regions that link the ellipsoid to the collagenous domains at both narrow ends of the ellipsoid), whereas the equatorial layers that connect both protomers front-to-front are formed by the six alternating II

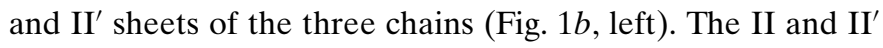
sheets provide a clipping mechanism that glues the three subunits of each protomer together (Fig. 1b). Each II or II' sheet is composed of four strands of one subdomain and of two strands forming a $\beta$-hairpin projected from the corresponding sheet of the adjacent subdomain or subunit (the topologies of the II and II' sheets are $\beta 4 \beta 3 \beta 8 \beta 6^{\prime} \beta 7^{\prime} \beta 9$ and $\beta 4^{\prime} \beta 3^{\prime} \beta 8^{\prime} \beta 6 \beta 7 \beta 9^{\prime}$, respectively; Fig. $\left.1 b\right)$. Thus, the $\beta 6^{\prime} \beta 7^{\prime}$ hairpin of sheet II corresponds to $\mathrm{C}$-sub of the same subunit and the $\beta 6 \beta 7$ hairpin of sheet $\mathrm{II}^{\prime}$ to $\mathrm{N}$-sub of the adjacent subunit.

\subsection{Structures of the $\alpha 1 \mathrm{NC} 1$ homohexamer and of an unreported $a 3 \mathrm{NC} 1$ homohexamer}

In vitro studies have shown that $\alpha 1 \mathrm{NC} 1$ can assemble into homohexamers (Khoshnoodi, Sigmundsson et al., 2006). We grew crystals of self-assembled recombinant $\alpha 1 \mathrm{NC} 1$ subunits
(Supplementary Fig. S1) that diffracted X-rays to $1.8 \AA$ resolution (Table 1). The asymmetric unit contained a homohexamer $\left(\alpha 1 \mathrm{NC1}_{\text {homo }}\right)$ with the same 'canonical' architecture as $\alpha 121 \mathrm{NC} 1$ and $\alpha 5 \mathrm{NC}_{\text {homo }}$ (Fig. 2, Supplementary Fig. S3b). We also obtained crystals of an $\alpha 3 \mathrm{NC} 1$ homohexamer that diffracted X-rays to $2.7 \AA$ resolution (Table 1 ), revealing canonical homohexamers $\left(\alpha 3 \mathrm{NC1}_{\text {homo }}\right.$; Fig. 2 , Supplementary Fig. S3b). Although $\alpha 3 \mathrm{NC} 1$ homohexamers were not observed by size-exclusion chromatography (Supplementary Fig. S2a), plasmon resonance studies (Supplementary Fig. S2b) showed self-association of $\alpha 3 \mathrm{NC} 1$ with a similar affinity as $\alpha 5 \mathrm{NC} 1\left(K_{\mathrm{d}}\right.$ values of 1.71 and $4.92 \mu M$, respectively; Supplementary Fig. $\mathrm{S} 2 b$ ) or $\alpha 1 \mathrm{NC1}$ (Khoshnoodi, Sigmundsson et al., 2006). These self-affinities, the canonical architectures of $\alpha 1 \mathrm{NC1}_{\text {homo, }}$, $\alpha 3 \mathrm{NC1}_{\text {homo }}$ and $\alpha 5 \mathrm{NC1}_{\text {homo, }}$, and the recent discovery of biologically formed $\alpha 5 \mathrm{NC1}_{\text {homo }}$ (Revert et al., 2018) indicate that either $\alpha 1 \mathrm{NC}_{\text {homo }}$ and $\alpha 3 \mathrm{NC1}_{\text {homo }}$ hexamers exist in vivo and have not been discovered to date or there are biological mechanisms that prevent their formation in vivo.

\subsection{Recombinant $\alpha 1 \mathrm{NC} 1$ and $\alpha 2 \mathrm{NC} 1$ chain mixtures reproduce the canonical naturally existing $\alpha 121 \mathrm{NC} 1$ hexamer}

We validated the use of self-assembled recombinant NC1 domains to reflect natural structures by generating the same $\alpha 121 \mathrm{NC} 1$ hexamer as previously isolated from BMs (Sundaramoorthy et al., 2002; Than et al., 2002; Vanacore et al., 2009) from a 2:1 mixture of pure $\alpha 1 \mathrm{NC} 1$ and $\alpha 2 \mathrm{NC} 1$ chains (Supplementary Fig. S1). Crystals of recombinant $\alpha 121 \mathrm{NC} 1$ diffracted X-rays to $2.8 \AA$ resolution (Table 1) and had the same architecture and folding (Fig. 2, Supplementary Fig. S3c) as the $\alpha 121 \mathrm{NC} 1$ hexamer isolated from BMs (the r.m.s.d. for the superimposition of all $\mathrm{C}^{\alpha}$ atoms of the hexamer is $0.48 \AA$; Supplementary Table S3). The recombinant $\alpha 121 \mathrm{NC} 1$ hexamer was composed of two identical protomers formed by two $\alpha 1 \mathrm{NC} 1$ domains and one $\alpha 2 \mathrm{NC} 1$ domain (Supplementary Figs. S4a, S4b and S4c). Each individual chain (designated $\alpha 1 \mathrm{NC}_{\alpha 121}$ and $\alpha 2 \mathrm{NC}_{\alpha 121}$ ) had an identical fold and relations to its natural counterpart (Supplementary Figs. S3c and S4; Supplementary Table S4; Than et al., 2002; Sundaramoorthy et al., 2002; Vanacore et al., 2004). Thus, recombinant NC1 chains seem to be appropriate for the formation and structural characterization of collagen IV NC1-domain assemblies. Therefore, the covalent modifications and/or cross-links found in natural $\alpha 121 \mathrm{NC} 1$ hexamers or potential directive roles of the collagenous domains or other macromolecular components do not seem to be essential for the correct self-assembly of $\alpha 121 \mathrm{NC} 1$.

\subsection{Structures of self-assembled $\alpha 4 \mathrm{NC} 1$ and $\alpha 2 \mathrm{NC} 1$ oligomers reveal possibly informative noncanonical homo-oligomers}

Given the lack of structural information on $\alpha 4 \mathrm{NC} 1$, we grew crystals of this chain alone that diffracted X-rays to $2.8 \AA$ resolution (Table 1). Surprisingly, they revealed a noncanonical hexameric protomer that generated a dodecamer 
$\left(\alpha 4 \mathrm{NC1}_{\text {homo }}\right)$ with an almost spherical shape upon application of the crystal symmetry (Fig. 2, Supplementary Fig. S5). The protomer resembled a canonical trimeric protomer, except that it had six subunits surrounding the molecular symmetry axis (Fig. 2), while the dodecamer resembled a canonical hexamer except for the increased number of subunits, resulting in a wider equatorial circumference $(\sim 100 \AA$ diameter $)$ and a widened central pole-to-pole tunnel $(\sim 40 \AA$ diameter versus $\sim 17 \AA$ in canonical homohexamers) (Fig. 2, Supplementary Fig. S5).

We also crystallized and determined the structure of the isolated $\alpha 2 \mathrm{NC} 1$ subunit at $2.5 \AA$ resolution (Table 1 ). The asymmetric unit included two subunits. Following application of the crystal symmetry, each subunit formed a protomer with fourfold molecular symmetry. One of these protomers was isolated and the other generated a homo-octamer $\left(\alpha 2 \mathrm{NC}_{\text {homo; }}\right.$;

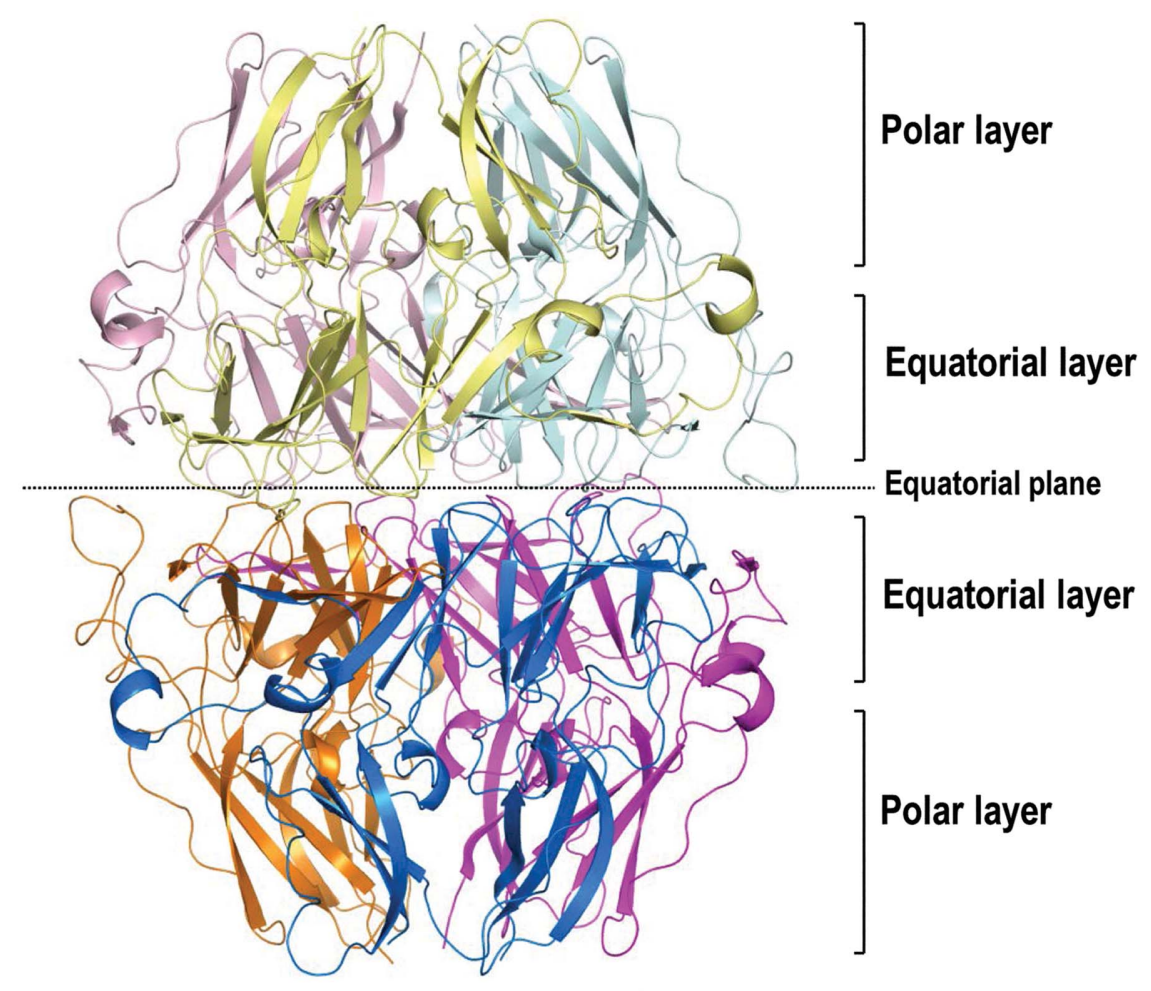

(a)

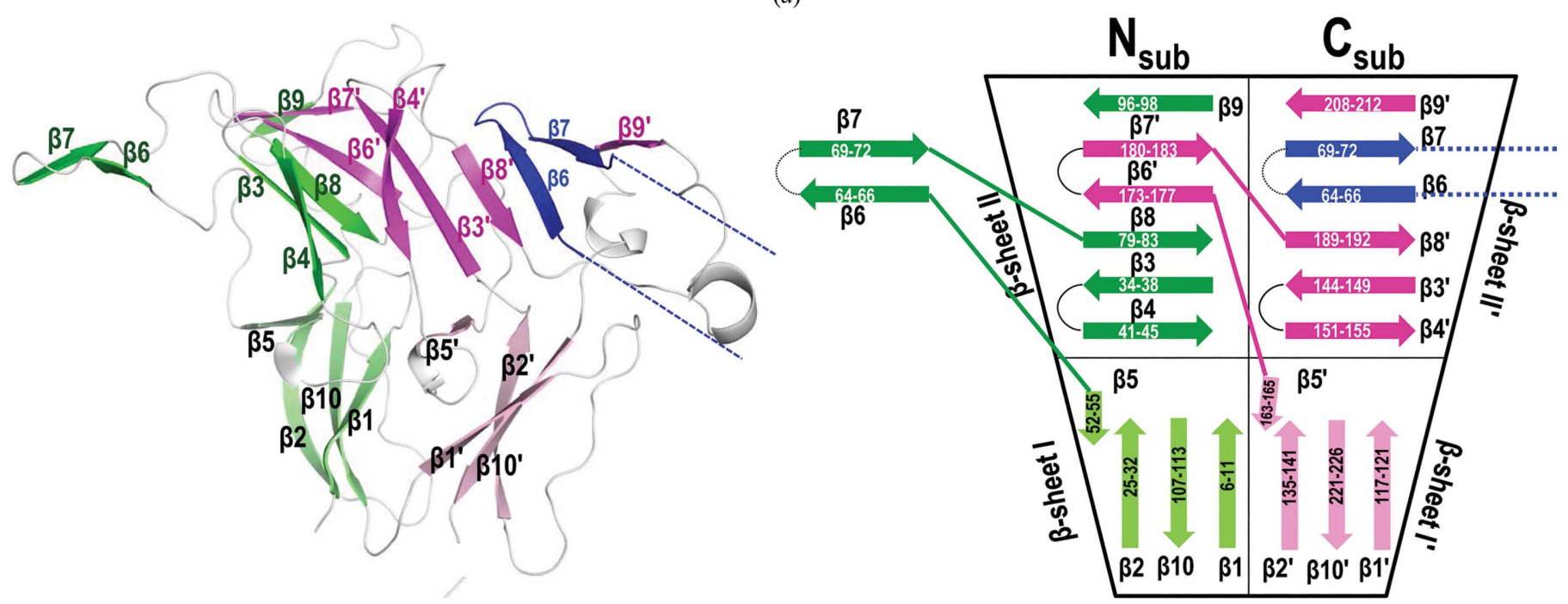

(b)

\section{Figure 1}

Crystal structure of $\alpha 5 \mathrm{NC}_{\text {homo }}$ (a) Cartoon representation of the crystal structure of recombinant $\alpha 5 \mathrm{NC} 1$ formed by a hexamer composed of two trimers. Each trimer contains three subunits of $\alpha 5 \mathrm{NC1}$ coloured magenta/orange, cyan/pink and blue/yellow. (b) Cartoon representation of a subunit with a schematic representation of the $\mathrm{N}$-subdomain (green) and $\mathrm{C}$-subdomain (magenta) folds. The $\mathrm{N}$-subdomain consists of $\beta$-sheets I ( $\beta 1, \beta 2, \beta 5$ and $\beta 10)$ and II $\left(\beta 3, \beta 4, \beta 6^{\prime}, \beta 7^{\prime}, \beta 8\right.$ and $\left.\beta 9\right)$, while the C-subdomain consists of $\beta$-sheets $\mathrm{I}^{\prime}\left(\beta 1^{\prime}, \beta 2^{\prime}, \beta 5^{\prime}\right.$ and $\left.\beta 10^{\prime}\right)$ and $\mathrm{II}^{\prime}\left(\beta 3^{\prime}, \beta 4^{\prime}, \beta 6, \beta 7, \beta 8^{\prime}\right.$ and $\left.\beta 9^{\prime}\right)$. The scheme highlights the fact that $\beta 6$ and $\beta 7$ from another protomer (coloured blue) are swapped between the NC1 monomers, whereas $\beta 6^{\prime}$ and $\beta 7^{\prime}$ (coloured magenta) are swapped from the C-subdomain into the $\mathrm{N}$-subdomain of the same subunit. 

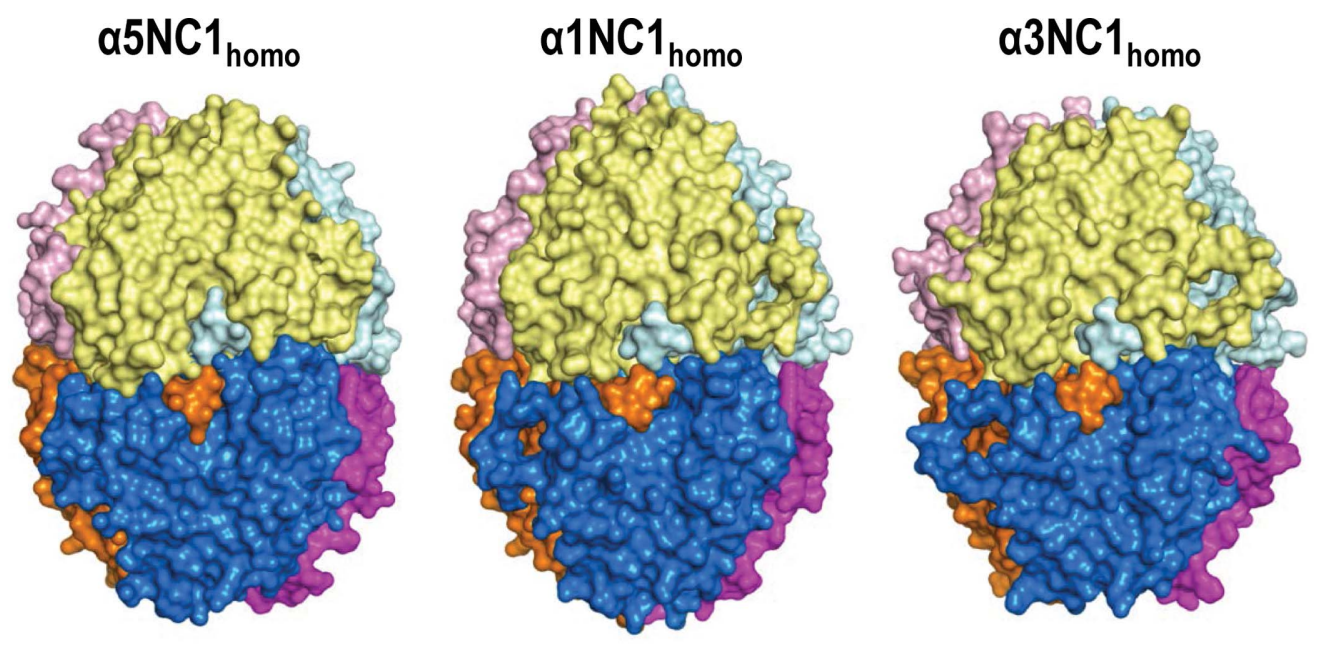

a121NC1
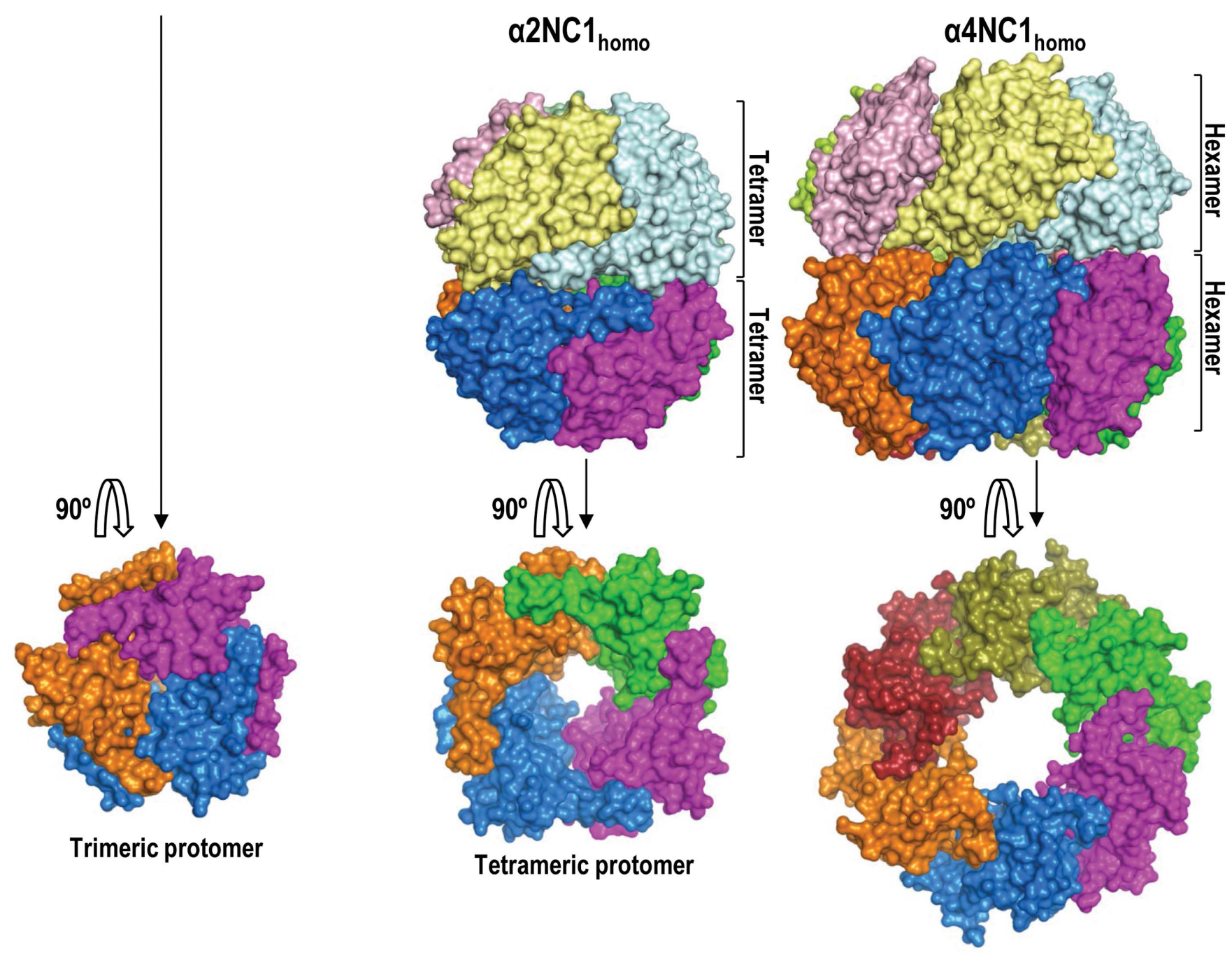

Hexameric protomer

Figure 2

Surface representations of the quaternary structures of the canonical hexameric $\alpha 5 \mathrm{NC}_{\text {homo }}, \alpha 1 \mathrm{NC}_{\text {homo, }}, \alpha 3 \mathrm{NC} 1_{\text {homo }}$ and $\alpha 121 \mathrm{NC} 1$ and the noncanonical $\alpha 2 \mathrm{NC}_{\text {homo }}$ and $\alpha 4 \mathrm{NC1}_{\text {homo }}$ organized as octamers and dodecamers, respectively. Each subunit in the assemblies is coloured differently. The top and middle rows show views in which the axis of highest molecular symmetry is vertical. In the bottom row this axis is perpendicular to the paper, allowing a view of the equatorial surface of the protomer. 
Fig. 2, Supplementary Fig. S5). $\alpha 2 \mathrm{NC}_{\text {homo }}$ resembled a canonical hexamer, except for an increase in the number of subunits to four per protomer, enlarging the equatorial circumference somewhat ( $\sim 70 \AA$ diameter) and widening the central tunnel $(\sim 30 \AA)$.

These two noncanonical protomers could be artifactual owing to the lack of the restrictions imposed by the triplehelical collagenous domain that is present in the complete collagen IV protomer. However, the protomers observed here in $\alpha 1 \mathrm{NC}_{\text {homo }}, \alpha 3 \mathrm{NC}_{\text {homo }}, \alpha 5 \mathrm{NC}_{\text {homo }}$ and $\alpha 121 \mathrm{NC} 1$ were trimeric despite the absence of a collagenous triple helix. Therefore, the two self-assembled noncanonical structures of $\alpha 2 \mathrm{NC} 1$ or $\alpha 4 \mathrm{NC} 1$ subunits may lack some structural elements that are present in the canonical trimeric protomers that could intrinsically restrict the formation of protomers with more than three subunits. The comparison between the structure of the $\alpha 2 \mathrm{NC} 1$ domain in its noncanonical homohexamer and the same domain in $\alpha 121 \mathrm{NC} 1\left(\alpha 2 \mathrm{NC}_{\alpha 121}\right)$ revealed that there are conformational differences that might explain the greater number of subunits (Fig. 2, Supplementary Fig. S6). Similar differences were observed when $\alpha 4 \mathrm{NC} 1$ was compared with the NC1 domains in the canonical hexamers (Fig. 3, Supplementary Fig. S6). These differences affected $\beta$-sheets II and II' exclusively. These two sheets ensure the clipping of the protomer into a closed trimeric structure and form the equatorial layer that connects both protomers front-to-front. Here, we define three structural motifs in sheet II (Fig. 3), SM1 (hairpin $\beta 3-\beta 4$ ), SM2 (hairpin $\beta 6-\beta 7$ ) and SM3 ( $\beta 9$ and the preceding loop), and their homologous counterparts in sheet $\mathrm{II}^{\prime}, \mathrm{SM} 1^{\prime}$ (hairpin $\beta 3^{\prime}-\beta 4^{\prime}$ ), SM2' (hairpin $\beta 6^{\prime}-\beta 7^{\prime}$ ) and $\mathrm{SM} 3^{\prime}$ ( $\beta 9^{\prime}$ and the preceding loop) (Figs. $3 a$ and $3 b$ ).

As can be seen in the canonical structures, SM1 and SM1' (Fig. $3 b$ ) face the central tunnel, creating a closed 'barrel'-like arrangement of six alternating $\beta 4$ and $\beta 4^{\prime}$ strands, which allows compact intertwining of three monomers (Than et al., 2002). These hairpins (residues $37-45$ in the case of SM1 and residues $144-154$ in the case of $\mathrm{SM}^{\prime}$ ) were not visible in the structure of $\alpha 2 \mathrm{NC1}_{\text {homo }}$ (Fig. 3), as would be expected if they were disordered in this noncanonical homo-octamer. On the other hand, they were visible in $\alpha 4 \mathrm{NC1}_{\text {homo }}$ but with a different conformation to that in $\alpha 2 \mathrm{NC1}_{\alpha 121}$ (Fig. 3). These changes are expected to decrease the strength of the association of the subunits into the protomer.

As described above (Fig. 1), SM2 and SM2' are inserted into the equatorial layers of the adjacent subunit or subdomain, respectively. In $\alpha 2 \mathrm{NC1}_{\text {homo }} \mathrm{SM} 2$ underwent a rigid-body rotation of $22.5^{\circ}$ (calculated by DYNDOM; Hayward \& Berendsen, 1998) relative to its position in $\alpha 2 \mathrm{NC1}_{\alpha 121}$, while in $\alpha 4 \mathrm{NC}_{\text {homo }} \mathrm{SM} 2$ adopted a random-coil conformation (Fig. 3, Supplementary Fig. S6). These alterations could weaken the closing belt that would have contributed to restricting the number of subunits to three in a canonical protomer.

The SM3' motif (Figs. $3 a$ and $3 b$ ) flanks SM2 peripherally (relative to the molecular symmetry axis of the protomer). $\mathrm{SM}^{\prime}$ was disordered and was not visible in either $\alpha 2 \mathrm{NC}_{\text {homo }}$ or $\alpha 4 \mathrm{NC}_{\text {homo }}$ (Fig. 3), again weakening the insertion of SM2 into the $\mathrm{II}^{\prime}$ sheet and thus the clipping together of the oligomer. In fact, the loop connecting SM2 to $\beta 8$ ( $\mathrm{L} \beta 7 \beta 8$; residues $62-77$ ), as well as the homologous loop connecting strands $\beta 7^{\prime}$ and $\beta 8^{\prime}$ ( $\mathrm{L} \beta 7^{\prime} \beta 8^{\prime}$; residues $\left.185-189\right)$ in sheet $\mathrm{II}^{\prime}$, also adopted alternative arrangements relative to the canonical structures (Fig. $3 c$ ) in $\alpha 2 \mathrm{NC1}_{\text {homo }}$ and $\alpha 4 \mathrm{NC1}_{\text {homo. Given }}$ the closeness of the $\mathrm{L} \beta 7 \beta 8$ and $\mathrm{L} \beta 7^{\prime} \beta 8^{\prime}$ loops to the SM2 ${ }^{\prime}$ and SM2 motifs, which are inserted between strands $\beta 8$ and $\beta 9$ of the two homologous sheets II $^{\prime}$ and II, these conformational changes should weaken the restricting belt that helps compel the protomer to be trimeric.

In addition to playing a key role in the formation of the protomer, all six SMs, as well as loops $\mathrm{L} \beta 7 \beta 8$ and $\mathrm{L} \beta 7^{\prime} \beta 8^{\prime}$, play crucial roles in the front-to-front interactions through the equatorial plane that glue two protomers together to generate the final particle (see the top row in Fig. $3 b$ ). Loop $\mathrm{L} \beta 7 \beta 8$ is the chloride-binding motif $\left(\mathrm{Cl}_{\mathrm{A}}\right.$ motif $)$ that assists protomerprotomer interaction across the equatorial plane following chloride binding (Cummings et al., 2016; Fig. 4a). The rearrangements and structural alterations in these elements observed in $\alpha 2 \mathrm{NC}_{\text {homo }}$ and $\alpha 4 \mathrm{NC}_{\text {homo }}$ (Fig. 3) modify the equatorial plane of the tetrameric and hexameric protomers, abolishing most of the protomer-protomer interactions that are observed in the canonical hexamer. However, the persistence of some interactions through the equatorial plane can explain the association of the two noncanonical protomers of $\alpha 2 \mathrm{NC} 1$ and $\alpha 4 \mathrm{NC} 1$ into octamers and dodecamers, respectively, which overall follow the general plan of the canonical hexameric assembly. The presence of an isolated tetrameric protomer that is not associated into an octamer in the $\alpha 2 \mathrm{NC}_{\text {homo }}$ crystal is structural evidence of weakened interactions across the equatorial plane.

Overall, a comparison of noncanonical and canonical oligomers (Figs. $3 a$ and $3 b$ ) revealed that the SM1, SM1' and SM2 flexible regions are key determinants in protomer formation, while the loops connecting these motifs, $\mathrm{L} \beta 7 \beta 8$ (motif $\mathrm{Cl}_{\mathrm{A}}$ ), $\mathrm{L} \beta 7^{\prime} \beta 8^{\prime}$ and $\mathrm{SM} 3^{\prime}$, are needed to establish the protomer-protomer interactions that generate the hexamer. It is interesting that among these structurally more variable regions (Fig. $3 c$ ), the loops that connect $\beta 8$ and $\beta 9$ (L $\beta 8 \beta 9$ ), $\beta 6^{\prime}$ and $\beta 7^{\prime}\left(\mathrm{L} \beta 6^{\prime} \beta 7^{\prime}\right)$, and $\beta 8^{\prime}$ and $\beta 9^{\prime}\left(\mathrm{L} \beta 8^{\prime} \beta 9^{\prime}\right)$ present length differences of one or two residues among the various types of NC1 chains (see below; Fig. 5b), suggesting that these regions provide structural variability to the oligomers. Indeed, previous work with the natural $\alpha 121 \mathrm{NC} 1$ heterohexamer has reported structural inter-chain variability in the conformations of these loops (Sundaramoorthy et al., 2002; Than et al., 2002). Here, these loops show variations in the structures of our canonical homohexamers (Supplementary Table S4 and Supplementary Fig. S7).

In summary, mobility and conformational changes are concentrated in the equatorial layer formed by $\beta$-sheets II and $\mathrm{II}^{\prime}$, a layer that is crucial for clipping the structure of the protomer and that connects both protomers front-to-front, whereas sheets I and I' of the polar layer exhibit high structural conservation in canonical and noncanonical protomers (Fig. 3c). In the polar layer only the loop between $\beta 1$ and $\beta 2$ exhibited substantial movement (translated into higher values 

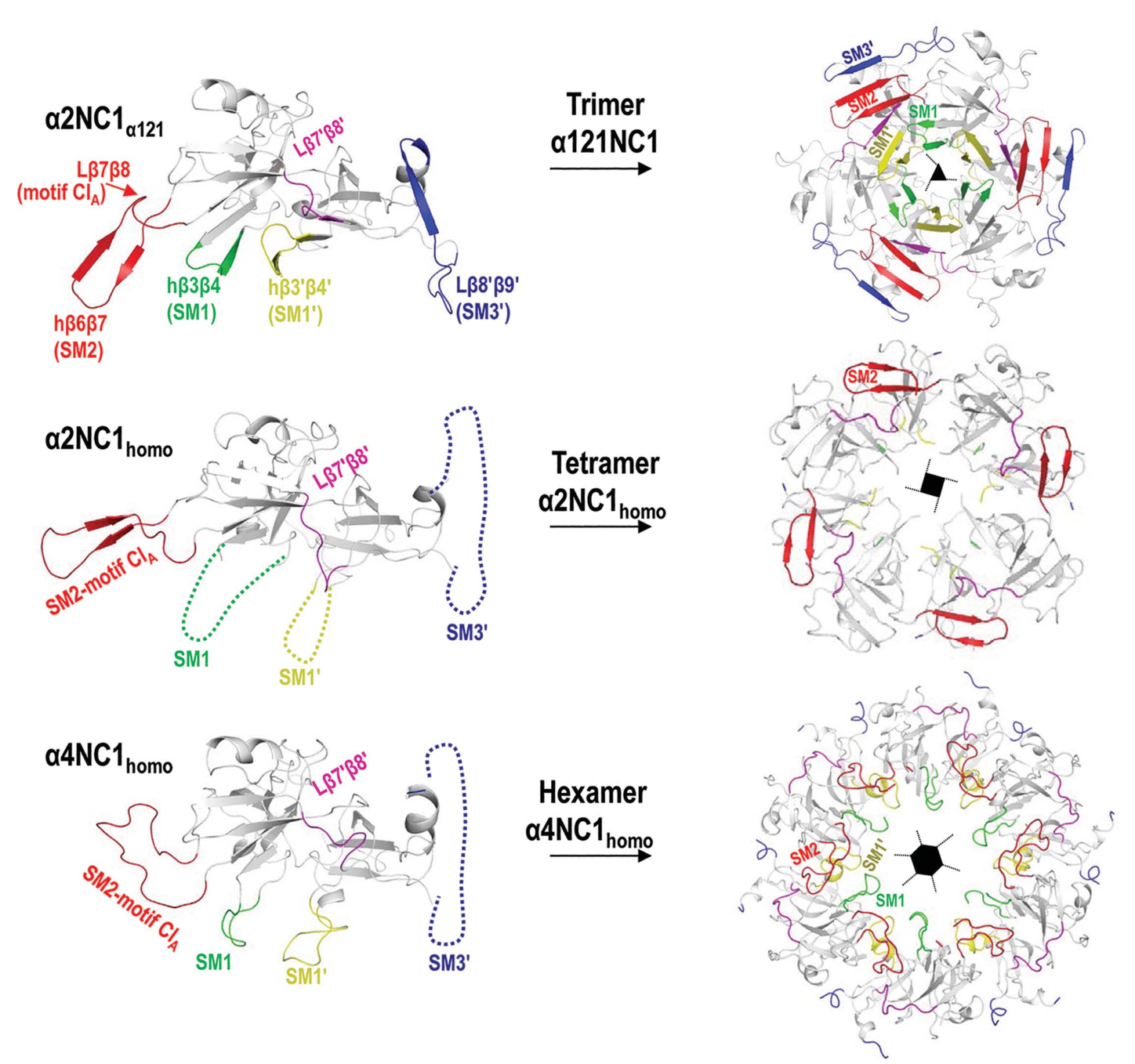

(a)
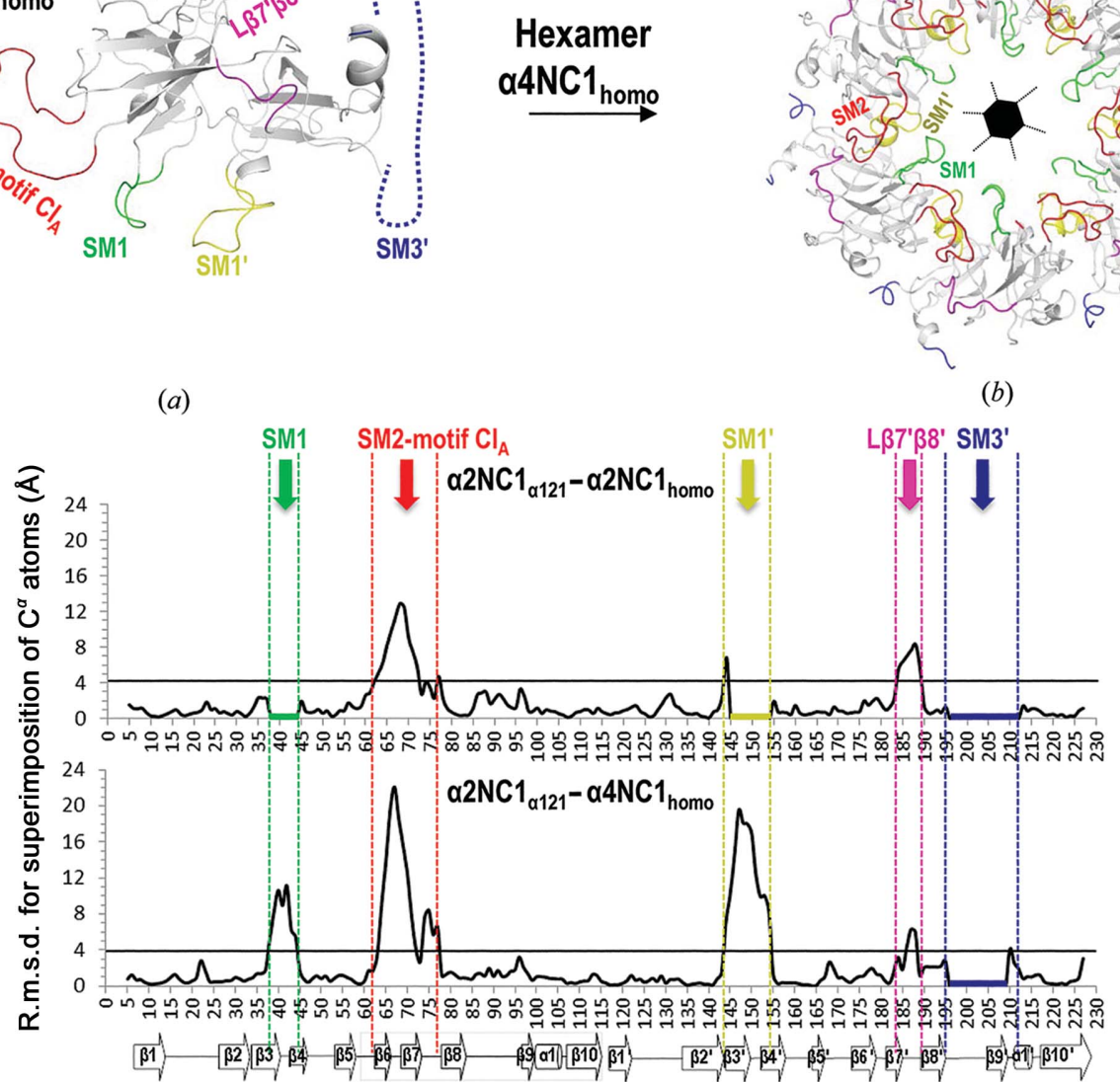

(c)

Figure 3

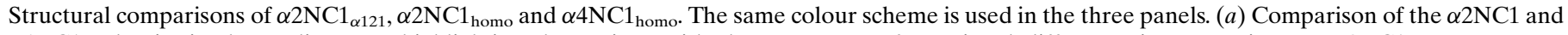
$\alpha 4 \mathrm{NC1}$ subunits in these oligomers, highlighting the regions with the greatest conformational difference in comparison to $\alpha 2 \mathrm{NC1} 1_{\alpha 121}$. Important structural motifs (SMs; see text) are labelled and coloured. Dashed lines represent highly disordered regions that are not visible in the crystal structures. (b) Cartoon representation of the structures of an $\alpha 121 \mathrm{NC} 1$ protomer and the corresponding noncanonical $\alpha 2 \mathrm{NC} 1_{\text {homo }}$ and $\alpha 4 \mathrm{NC} 1_{\text {homo }}$ tetrameric and hexameric protomers. (c) Plot of r.m.s.d. deviation per $\mathrm{C}^{\alpha}$ atom along the sequences between $\alpha 2 \mathrm{NC}_{\alpha 121}$ and $\alpha 2 \mathrm{NC}_{\text {homo }}$ (top) and $\alpha 4 \mathrm{NC} 1_{\text {homo }}$ (bottom). Dashed vertical lines enclose the structural motifs (identified with arrows) showing high r.m.s.d.s. The thick horizontal line (residues 196-210) indicates a lack of electron density. 
for the r.m.s.d. between odd-numbered and even-numbered NC1 chains; Supplementary Fig. S7), caused by a short flapping movement $\left(\sim 30^{\circ}\right)$ with no alteration of the actual structure of this loop, leading only to changes in its position. Given their high structural stability, sheets I and I' may form the initial structural core of the subunit, folding first, while the flexible regions of $\beta$-sheets II and II' could be involved in the termination of the folding process, ensuring proper protomer assembly. At this point, we are unable to identify the reasons for the insertion of just one extra subunit or of three extra subunits in the $\alpha 2 \mathrm{NC} 1$ and $\alpha 4 \mathrm{NC} 1$ protomers, respectively. However, it seems clear that the disorder or loss of the SM1 and SM1' secondary structure observed in the noncanonical $\alpha 2 \mathrm{NC}_{\text {homo }}$ and $\alpha 4 \mathrm{NC}_{\text {homo }}$ protomers, which leads to loss of

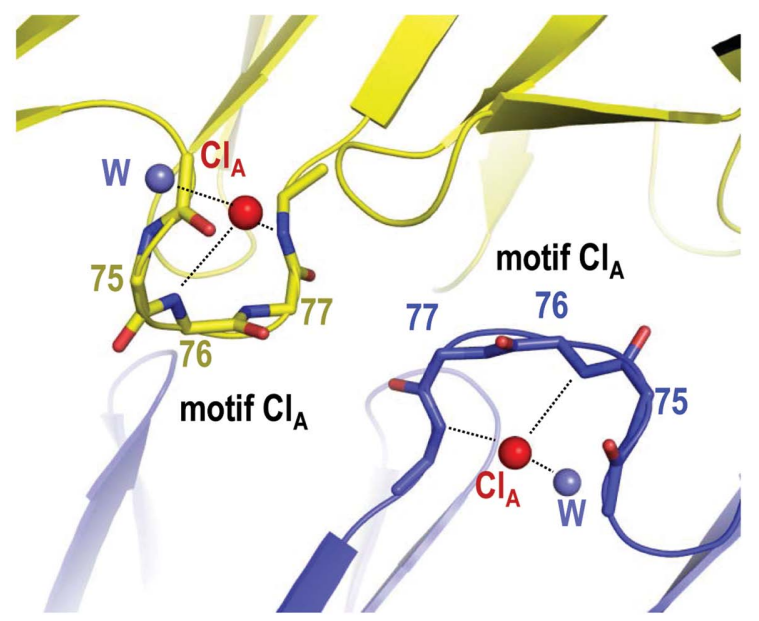

$(a)$

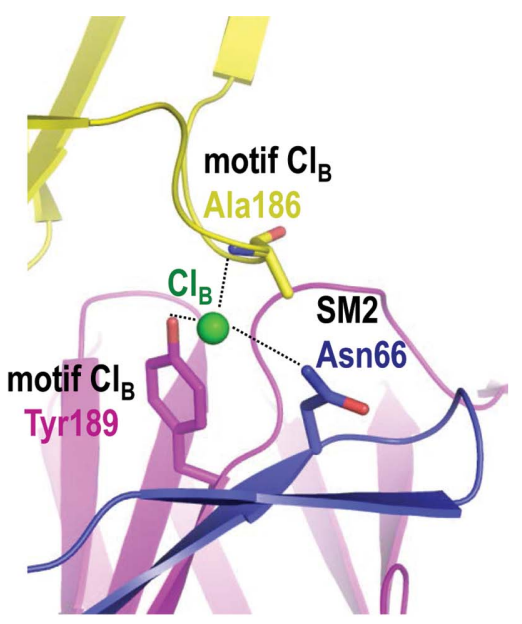

(b)

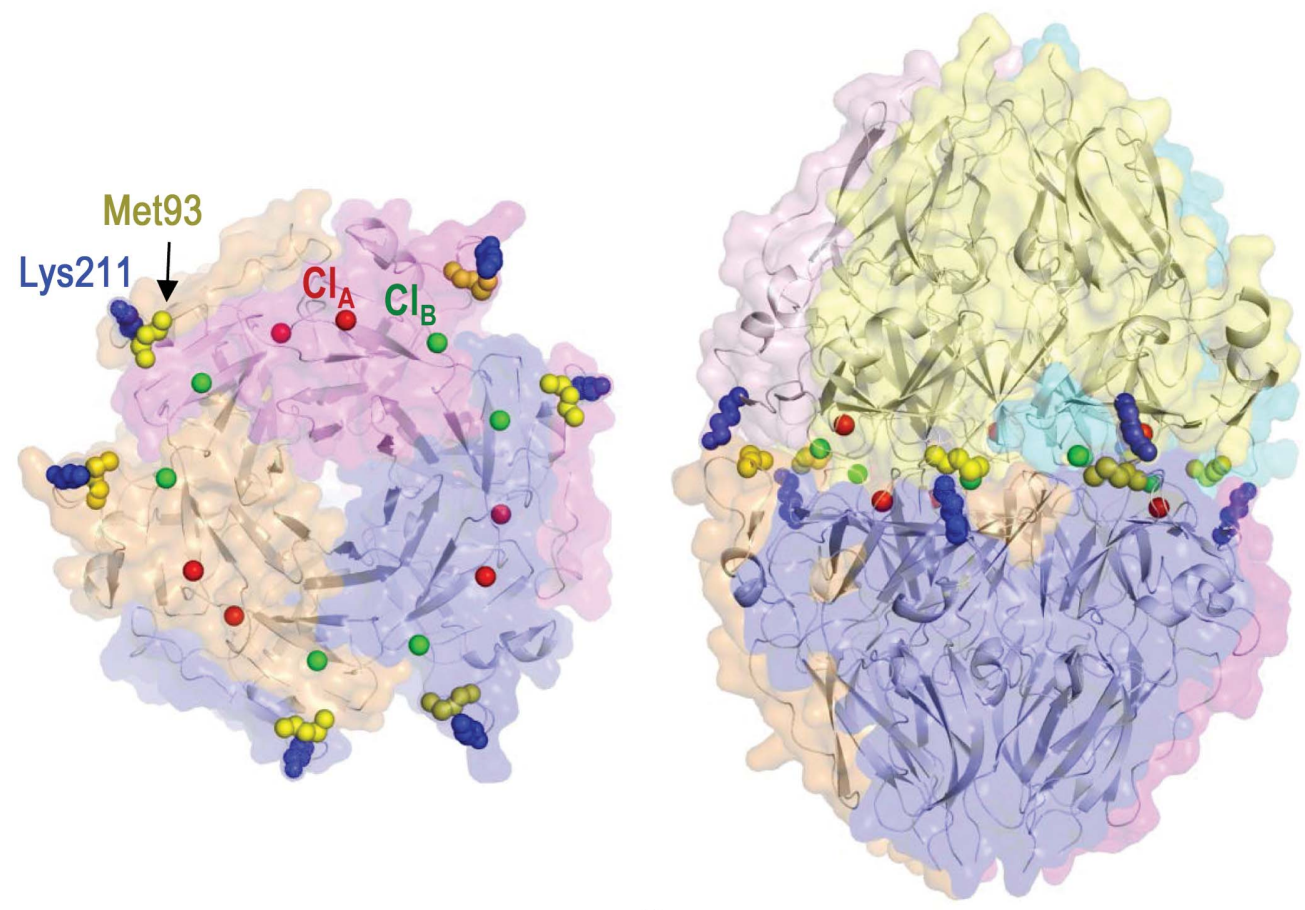

(c)

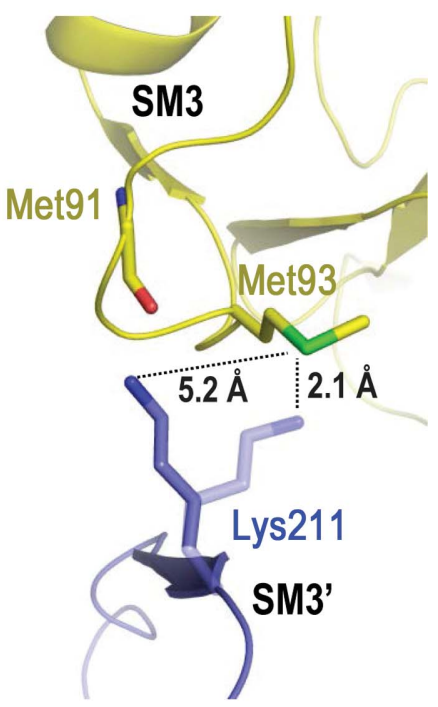

$(d)$

Figure 4

Hexamer stabilization through ion binding and potential sulfilimine-bond sites. (a) The chloride ion $\mathrm{Cl}_{\mathrm{A}}$ (red) interacts with the main-chain amino groups of $\mathrm{L} \beta 7 \beta 8\left(\mathrm{Cl}_{\mathrm{A}}\right.$ motif) and a water molecule (blue) in two opposing monomers. $(b)$ The chloride ion $\mathrm{Cl}_{\mathrm{B}}$ (green) binds to residues of flexible regions from three opposing monomers in the $\mathrm{Cl}_{\mathrm{B}}$ motif (Ala186 in $\mathrm{L} \beta 7^{\prime} \beta 8^{\prime}$ and Tyr189 in $\beta 8^{\prime}$ ) and Asn66 in SM2. (c) Semi-transparent surface representation (enclosing a cartoon representation) of the equatorial view of the protomer in the canonical $\alpha 1 \mathrm{NCl}_{\text {homo }}$ hexamer indicating the six $\mathrm{Cl}_{\mathrm{A}}$ ions (red) and six $\mathrm{Cl}_{\mathrm{B}}$ ions (green) at the interface between the protomers in the hexamer. In addition, the six interfacial Met 93 residues (yellow) and the six Lys211 residues (blue), three from the shown protomer and the other three from the top protomer (not shown), are also indicated to stress their proximity, which allows easy sulfilimine-bond formation. These residues and the $\mathrm{Cl}_{\mathrm{A}}$ and $\mathrm{Cl}_{\mathrm{B}}$ ions are also shown in the view of the complete $\alpha 1 \mathrm{NC1} 1_{\text {homo }}$ hexamer illustrated on the right. (d) Zoom on the flexible $\mathrm{L} \beta 8 \beta 9$ (SM3) and $\beta 9^{\prime}$ (SM3') regions of opposing monomers, highlighting the closeness of the Met93 and Lys211 residues of each region, respectively. A mere rotamer change of Lys211 would place it in position for the formation of a sulfilimine bridge with Met93. 

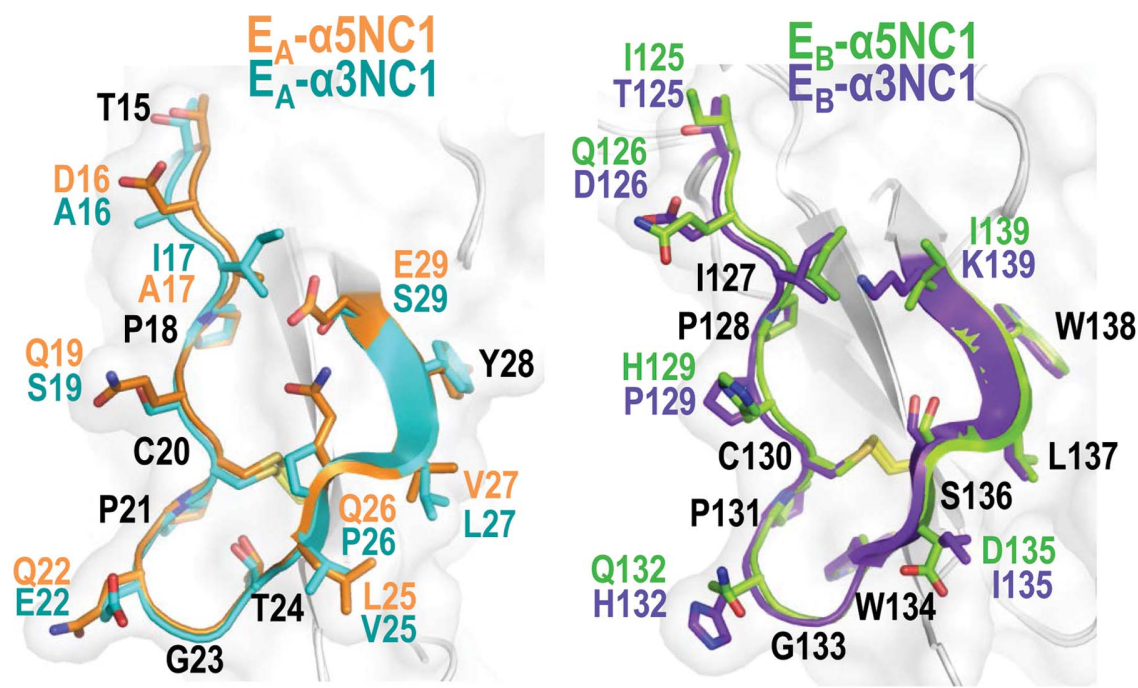

(a)

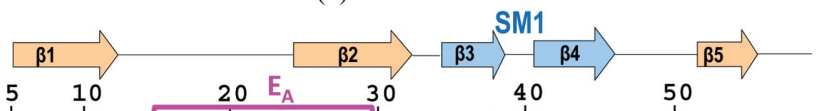

a1NC1 1 SVDHGFLVTRHSQTIDDP QCP SGTKI LYHGY SLLYVQGNERAHGQDLGTAGSCLRKFST 59 $\alpha 3 N C 111$ WTTRGFVFTRH SQTTA IP SCPEGTVPLYSGF SF LFV QGNQRAHGQDLGTLGSCLQRFTT 59 $\alpha 5 \mathrm{NC1} 11$ SVAHGFLITRHSQTTDAPQCP QGTLQVY EGF SLLYVQGNKRAHCQDLGTAGSCLRRFST 59

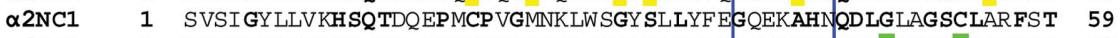

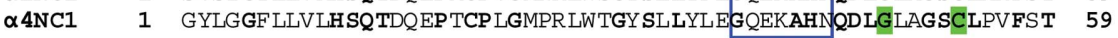
SM2 motif $\mathrm{Cl}_{A}$ $\beta \sqrt{-\beta 7}-\beta 9-\alpha 10-\beta 10-\beta 1>$

$\alpha 1 \mathrm{NC1} \quad 60$ MPFLFCNINNV CNFASRNDYS YWLST PE PMPMSMAP ITGEN IRPFI SRCAVCEAPAMVMA 119 $\alpha 3 N C 1 \quad 60$ MPFLFCNVNDV CNFAS RNDYSYWLSTPALMPMNMAP ITGRALEPYI SRCTVCEGPA IA IA 119 $\alpha 5 N C 1 \quad 60$ MPFMFCNINNVCNFAS RNDYS YWLST PE PMPMS MQP LKGQS IQPFI SRCAVCEAPA VVIA 119 $\alpha 2 N C 1 \quad 60$ MPFLYCNPGDVCYYAS RNDKS YWLSTTA PLPM- -MPVAEDE IKPYI SRCSVCEAPA IA IA 117 $\alpha 4 N C 1 \quad 60$ LPEA YCNIHQVCHYAQRNDRS YWLASAA PLPM- -MPLSEEA IR PYV SRCAVCEAPAQAVA 117

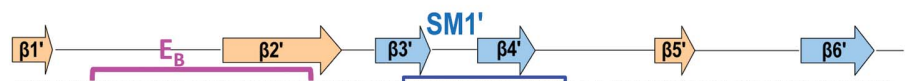

$\alpha 1 \mathrm{NC1} 120$ VHSQTIOIP PCPSGWS SLWIGYS FVMITSAGAEGSGOALAS PGSCLEEFRSAPFIECHG- 178 $\alpha 3 N C 1120$ VHSQTTDIP PCPHGWI SLWKG FS FIMFT SAGSE GTGQALAS PGSCL EE FRASP FLECHG- 178 $\alpha 5 \mathrm{NC1} 120$ VHSQTI QIPHCPQGWD SLWIGYS FMMUT SAGAE GSGOALAS PGSCL EE FRSAP FIECHG- 178 $\alpha 2 N C 1118$ VHSQDVSIPHCPAGWRSLWIGYSFLMHTAAGDEGGG S LVS PGSCLED FRA TPFIECNGG 177

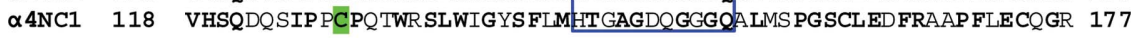
$\beta 7^{\prime}-\beta 3^{\prime}-\beta 9^{\prime}-\alpha 1^{\prime}-\beta 10^{\prime}$

Q1NC1 179 RGTCNYYANAYSFWLATI ERSEMFKKPTPSTLK-AGELRTHVSRCQVCMRRT 229 «3NC1 179 RGTCNYYSNSY SEWLASL-NPERMFRKP IPS TVK-AGELEKII SRCQVCMKKRH 230 $\alpha 5 N C 1 \quad 179 \quad$ RGTCNYYANSYSEWLATV-DVSDMFS KPQSETLK-AGDLRTRI SRCQVCMKRT 229 $\alpha 2 N C 1 \quad 178 \quad$ RGTCHYYANKY SEWLTTI PEQSFQGSP SADTLK-AGL IRTHI SRCQVCMKNL 228 $\alpha 4 N C 1 \quad 178$ QGTCHF EANKY SFWLTTVAADLQFSSAPAPDTLKESQAQRQKI SRCQVCVKYS 230

(b)

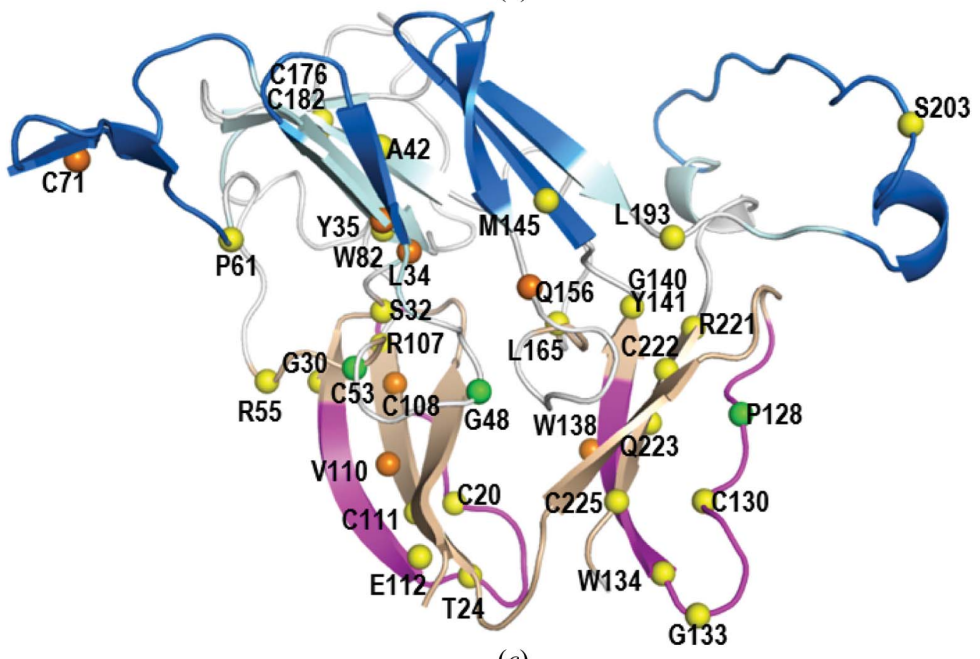

the $\beta$-barrel-like organization around the central tunnel, could affect SM2 and the $\mathrm{Cl}_{\mathrm{A}}$ motif of the same subunit, resulting in the displacement of these two elements away from the central tunnel. The structural disruption of SM1' could also affect the adjacent $\beta 8^{\prime}$ strand (which loses its secondary structure in the noncanonical protomers) and the insertion of SM2 in the next subunit (Fig. 3). SM2 is a key protomerclipping element that would become relaxed in the two noncanonical protomers, allowing the insertion of extra subunits. In turn, the resulting abnormal placement of SM2 could prevent interactions with SM3', which would avoid the generation of a narrow canonical protomer.

\subsection{Chloride-mediated protomer-} protomer stabilization and potential for sulfilimine cross-linking in canonical but not in noncanonical self-assembled oligomers of recombinant NC1 chains

The $\alpha 121 \mathrm{NC} 1$ structures obtained from natural sources showed the presence of ions and covalent sulfilimine bonds associated with stabilization of the hexamers (Vanacore et al., 2004; Than et al., 2002; Robertson et al., 2014). Chloride ions were also present in our $\alpha 1 \mathrm{NC1}_{\text {homo }}, \quad \alpha 3 \mathrm{NC1}_{\text {homo }}$ and $\alpha 5 \mathrm{NC1}_{\text {homo }}$ structures at two different positions in the protomer-protomer interface. Six chloride ions $\left(\mathrm{Cl}_{\mathrm{A}}\right)$, one per monomer,

Figure 5

Structural bases of Alport's and Goodpasture's syndromes. (a) Goodpasture's epitopes $\mathrm{E}_{\mathrm{A}}$ (left) and $E_{B}$ (right) as seen in the superimposed structures of subunits of $\alpha 3 \mathrm{NC1}_{\text {homo }}$ (cyan or blue) and $\alpha 5 \mathrm{NC} 1_{\text {homo }}$ (orange or green). The same colour code is used for the amino-acid side chains (except for invariant residues, which are shown in black). (b) Structure-assisted sequence alignment of the $\alpha 1-$ $5 \mathrm{NC} 1$ chains. Arrows indicate $\beta$-strands and cylinders indicate $\alpha$-helices. $\beta$-Strands of the $\mathrm{I} / \mathrm{I}^{\prime}$ and II/ II' sheets are coloured light pink and light blue, respectively. Blue rectangles enclose flexible regions and $\mathrm{Cl}$ motifs (labelled), horizontal magenta lines mark the $E_{A}$ and $E_{B}$ epitopes (also labelled) and coloured shadowing indicates the residues reported to host missense mutations in Alport's syndrome. (c) Alport's syndrome missense mutations (listed in Supplementary Table S5) are mapped onto the structure of an $\alpha 5 \mathrm{NC} 1$ subunit. Yellow spheres represent mutations in this chain, while superimposed mutations of $\alpha 3 \mathrm{NC1}$ and $\alpha 4 \mathrm{NC1}$ are coloured orange and green. $\beta$-Sheets $\mathrm{I} / \mathrm{I}^{\prime}$ and $\mathrm{II} / \mathrm{II}^{\prime}$ are coloured light pink and light blue, respectively, $E_{A}$ and $E_{B}$ are shown in magenta and flexible $S M$ regions and $\mathrm{Cl}$ motifs are presented in deep blue. 
were modelled at the position previously observed in the natural $\alpha 121 \mathrm{NC} 1$ structure (Figs. $4 a$ and $4 c$ ). $\mathrm{Cl}_{\mathrm{A}}$ interacted with the amino groups of the $\mathrm{Cl}_{\mathrm{A}}$ motif. In the structure of the $\alpha 121 \mathrm{NC} 1$ hexamer obtained from the BM of bovine lens capsule (Cummings et al., 2016), this motif was one of the flexible regions shown to be crucial for triggering the assembly of the two $\alpha 121 \mathrm{NC} 1$ protomers into a hexamer.

Six additional chloride ions $\left(\mathrm{Cl}_{\mathrm{B}}\right)$, again one per monomer, were also observed in the $\alpha 1 \mathrm{NC1}_{\text {homo }}, \alpha 3 \mathrm{NC}_{\text {homo }}, \alpha 5 \mathrm{NC}_{\text {homo }}$ and $\alpha 121 \mathrm{NC} 1$ hexamers (Figs. $4 b$ and $4 c$ ). In the $\alpha 121 \mathrm{NC} 1$ structure from the BM of bovine lens the nonprotein electron densities at these positions were interpreted as potassium ions, while in the equivalent $\alpha 121 \mathrm{NC} 1$ structures from the BMs of bovine and human placenta they were modelled as bromide ions and acetate, respectively (Vanacore et al., 2004). We modelled the densities at these positions as $\mathrm{Cl}^{-}$ions since the nature and geometry of the contacts mediated by them (with a hydroxyl group and with amino groups) and the electron densities fitted best with the presence of this anion, which is abundant in the crystallization drop. $\mathrm{Cl}_{\mathrm{B}}$ was located at the interprotomer interface and was involved in an interaction network between conserved residues that are present in $\mathrm{L} \beta 7^{\prime} \beta 8^{\prime}$ (hereafter referred to as the $\mathrm{Cl}_{\mathrm{B}}$ motif) and SM2 from three different monomers of opposing protomers (Fig. 4c). In contrast, in the noncanonical homoligomers $\alpha 2 \mathrm{NC1}_{\text {homo }}$ and $\alpha 4 \mathrm{NC1}_{\text {homo }}$ all chloride ions were missing mainly owing to the rearrangement of the $\mathrm{Cl}_{\mathrm{A}}$ and $\mathrm{Cl}_{\mathrm{B}}$ motifs as well as of SM2 and $\beta 8^{\prime}$ (Fig. 3), which prevented the coordination of these chlorides. Since all recombinant proteins used were prepared in $\mathrm{NaCl}$ buffers, and since hexamer assembly in the BM from bovine lens has recently been demonstrated to be chloridedependent (Cummings et al., 2016), the conformational changes observed in $\alpha 2 \mathrm{NC}_{\text {homo }}$ and $\alpha 4 \mathrm{NC1}_{\text {homo }}$ that prevent chloride binding may hinder the final protomer-protomer assembly.

As expected, in the final stage of network assembly the natural NC1 hexamers of collagen IV cross-linked by covalent sulfilimine bonds between Met93 from SM3 of one monomer and Lys211 from SM3' of another monomer facing across the equatorial plane were formed $(\alpha 1 \mathrm{NC} 1$ residue numbering; Than et al., 2002; Vanacore et al., 2009). The formation of the sulfilimine bonds is catalyzed by peroxidasin, an enzyme found in BMs (Bhave et al., 2012). No sulfilimine bonds were seen in the structures presented in this work, possibly because all of the proteins were produced in a recombinant system in which either the conditions were inadequate for the catalytic activity of peroxidasin or this enzyme was absent. However, in the $\alpha 1 \mathrm{NC}_{\text {homo }}, \alpha 3 \mathrm{NC1}_{\text {homo }}, \alpha 5 \mathrm{NC} 1_{\text {homo }}$ and $\alpha 121 \mathrm{NC} 1$ structures Lys211 interacts via a hydrogen bond with the SM3 containing Met93 of an opposing monomer (Figs. $4 c$ and $4 d$ ). An alternative conformation of the Lys211 side chain could allow sulfilimine-bond formation with Met93 (Fig. 4d). This was not the case for $\alpha 2 \mathrm{NC1}_{\text {homo }}$ and $\alpha 4 \mathrm{NC1} 1_{\text {homo }}$ (Fig. 3), where the inappropriate location of these residues for sulfilimine-bond formation reflects the high flexibility of $\beta 9^{\prime}$ in $\mathrm{SM}^{\prime}$, strongly suggesting that the structural elements involved in protomerprotomer assembly acquire their final conformation at the end the NC1 folding process. Thus, NC1 folding is correlated with the proposed monomer-protomer-hexamer sequential assembly model for collagen IV network formation (Boutaud et al., 2000; Kalluri, 2003; Khoshnoodi, Cartailler et al., 2006; Cummings et al., 2016).

\subsection{Insight into Alport's and Goodpasture's syndromes}

The $\alpha 345$ collagen IV network is a major component of the glomerular BM and its alteration underlies the pathogenesis of Alport's and Goodpasture's syndromes (Hudson et al., 2003; Pedchenko et al., 2010). In Alport's syndrome, mutations in these chains, including $\alpha 5 \mathrm{NC1}$, lead to a defective network assembly, causing the multi-laminar splitting of the glomerular BM. In Goodpasture's disease autoantibodies directed to the $\alpha 3 \mathrm{NC} 1$ and $\alpha 5 \mathrm{NC} 1$ domains are generated. Our structures of $\alpha 3 \mathrm{NC1}_{\text {homo }}, \alpha 4 \mathrm{NC1}_{\text {homo }}$ and $\alpha 5 \mathrm{NC1}_{\text {homo }}$ show that two structurally equivalent regions of $\alpha 3 \mathrm{NC}, \mathrm{E}_{\mathrm{A}}$ and $\mathrm{E}_{\mathrm{B}}$, as well as the homologous regions of $\alpha 5 \mathrm{NC1}$, encompass the Goodpasture epitopes (Figs. $5 a$ and $5 b$; Cui et al., 2016). Furthermore, the Alport missense mutations in the NC1 domains (Crockett et al., 2010; see Supplementary Table S5) predominantly map to the more structurally stable regions of the $\mathrm{NC1}$ domain ( $\sim 90 \%$ of the mutations), mainly $\beta$-sheets $\mathrm{I} / \mathrm{I}^{\prime}$, with few mutations mapping to those regions that showed high flexibility in $\alpha 2 \mathrm{NC}_{\text {homo }}$ and $\alpha 4 \mathrm{NC} 1_{\text {homo }}(\sim 10 \%$ of the mutations; Figs. $5 b$ and $5 c$ ). Since the structural core of the NC1 domains should acquire its conformation in the initial steps of NC1 folding (see Fig. 6 and Discussion) to nucleate protomer assembly, these mutations could strongly compromise collagen IV network formation. Supporting this idea, most of the Alport mutations $(68 \%)$ are found in residues with high structural relevance, such as cysteine, glycine or proline (Supplementary Table S5).

To our knowledge, the structures presented here represent the first visualization of the Goodpasture autoantigen. The regions that encompass the $\mathrm{E}_{\mathrm{A}}$ and $\mathrm{E}_{\mathrm{B}}$ epitopes lie in $\beta$-sheets $\mathrm{I} / \mathrm{I}^{\prime}$, a more structurally constant and fixed part of the NC1 structural core (Figs. $1 b$ and $5 c$ ). The comparison of $\mathrm{E}_{\mathrm{A}}$ and $\mathrm{E}_{\mathrm{B}}$ of $\alpha 3 \mathrm{NC} 1$ and $\alpha 5 \mathrm{NC} 1$ also showed that they are identical in structure and also to the corresponding part of $\alpha 1 \mathrm{NC} 1$, although no antigenicity has been determined in the latter chain in patients with Goodpasture's disease (Supplementary Figs. S8 and S9). Solvent-accessibility analysis of residues at the $\mathrm{E}_{\mathrm{A}}$ and $\mathrm{E}_{\mathrm{B}}$ epitopes in the $\alpha 3 \mathrm{NC} 1$ and $\alpha 5 \mathrm{NC} 1$ structures, in comparison to the corresponding residues in $\alpha 1 \mathrm{NC} 1$, showed similar exposed surface areas in either monomers or hexamers. Very small differences in solvent accessibility were observed for Leu27 and Tyr28 in $\mathrm{E}_{\mathrm{A}}$ and for Pro131 and Trp134 in $\mathrm{E}_{\mathrm{B}}$ (Supplementary Fig. S10). These residues are highly conserved among NC1 chains (Supplementary Figs. S8a and $\mathrm{S} 9 a$ ). They form a hydrophobic patch on the exposed surface of the protomer (Supplementary Fig. S11a). Further comparisons with the corresponding regions of $\alpha 2 \mathrm{NC1}_{\text {homo }}$ and $\alpha 4 \mathrm{NC1}_{\text {homo }}$ revealed conservation of the fold in this region even in these noncanonical oligomers (Supplementary Figs. S11b and S11c). Therefore, the differential antigenicity 
observed between chains must be owing to sequence determinants, as has recently been proposed (Cui et al., 2016).

\section{Discussion}

The very recent report of the existence of the $\alpha 5 \mathrm{NC} 1$ homohexamer in vivo (Revert et al., 2018) gives credence to the view that the canonical $\alpha 1 \mathrm{NC} 1$ and $\alpha 3 \mathrm{NC} 1$ homohexamers presented here may also exist in vivo, particularly given their structural stability, their proper binding of chloride ions for hexamer stabilization and their correct disposition of the residues that mediate the sulfilimine cross-linking bonds that stabilize the hexamer. Their spontaneous self-assembly in solutions of individual components, and even in mixtures of different components, attests to their thermodynamic stability and the absence of important kinetic barriers against their formation.

The finding that only a few combinations of collagen IV chain types are present in BMs, among the $>1000$ possible combinations of the six collagen IV chains, is difficult to explain based only on spontaneous thermodynamically driven assembly. Although the contribution of the collagenous parts to chain selection has not yet been clarified, our findings with isolated NC1 domains without the collagenous parts suggest the existence of regulatory mechanisms that could allow or guide the selection of a given chain combination. Spontaneous assembly has been proposed for $\alpha 121 \mathrm{NC} 1$, where the $\alpha 2 \mathrm{NC} 1$ chain shows a higher affinity to interact with $\alpha 1 \mathrm{NC} 1$ than with itself (Khoshnoodi, Sigmundsson et al., 2006). In fact, we show here that 2:1 mixtures of $\alpha 1 \mathrm{NC} 1$ and $\alpha 2 \mathrm{NC} 1$ chains spontaneously generate the natural $\alpha 121 \mathrm{NC} 1$ heterohexamer. Conversely, our experimental results have shown that equimolecular mixtures of $\alpha 3 \mathrm{NC} 1, \alpha 4 \mathrm{NC} 1$ and $\alpha 5 \mathrm{NC} 1$ only produce $\alpha 5 \mathrm{NC}_{\text {homo }}$ crystals. Therefore, further studies will be required to clarify the mechanism underlying the alternative formation of $\alpha 5$ and $\alpha 345$ networks in vivo.

Our findings of noncanonical assemblies for $\alpha 2 \mathrm{NC} 1$ and $\alpha 4 \mathrm{NC} 1$ homo-oligomers and the structural changes observed in the chains forming these noncanonical protomers in comparison to $\alpha 2 \mathrm{NC}_{\alpha 121}$ and to the chains in $\alpha 1 \mathrm{NC}_{\text {homo, }}$, $\alpha 3 \mathrm{NC1}_{\text {homo }}$ and $\alpha 5 \mathrm{NC1}_{\text {homo }}$ could imply that chain folding is closely related to protomer assembly. It is unlikely that

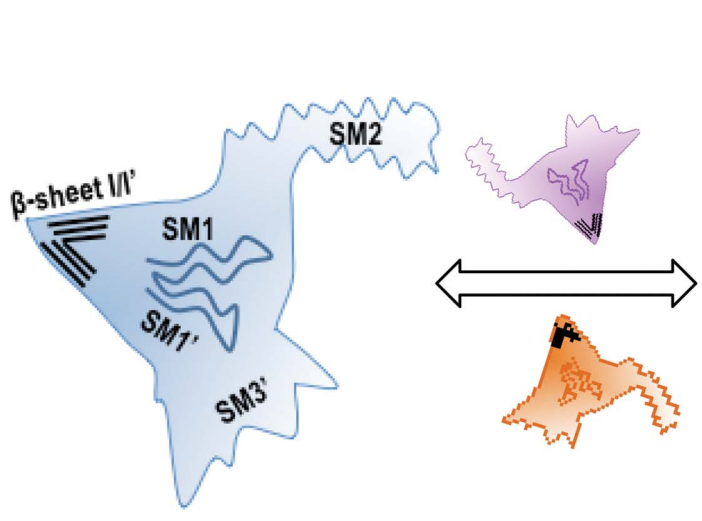

(a)

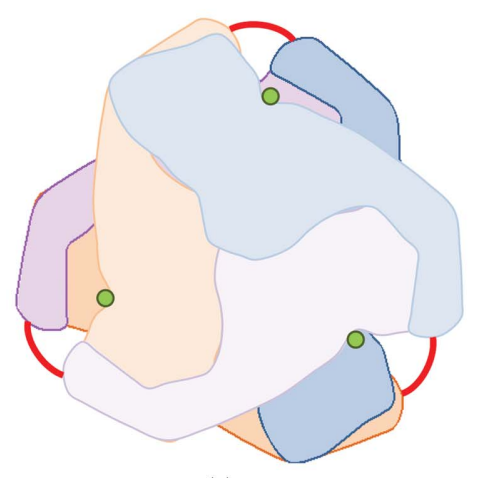

(c)

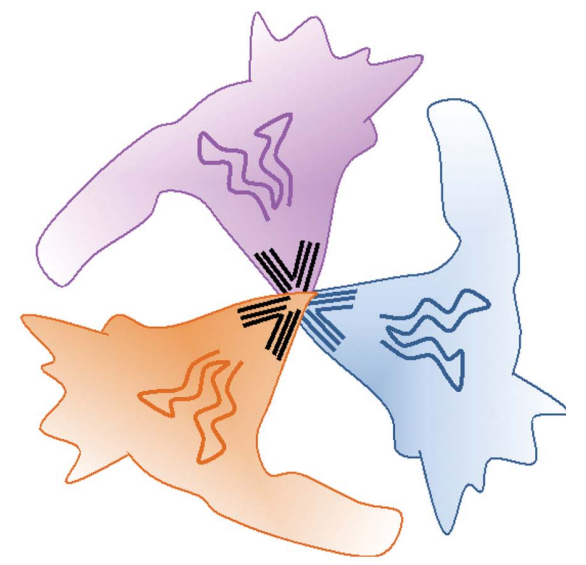

(b)

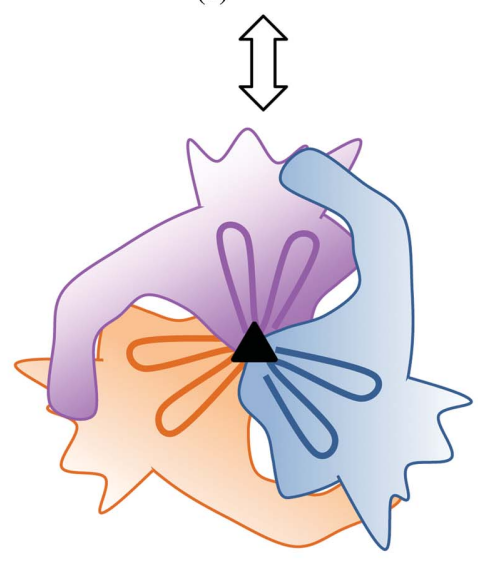

$(d)$

Figure 6

Proposed model for canonical hexamer assembly. Individual monomers $(a)$ start to nucleate a protomer via $\beta$-sheets I/I' $(b)$. Next, the SM1/1' and SM2/SM2' flexible regions from $\beta$-sheets II/II' are stabilized in the nascent protomer, resulting in favoured additional intersubunit interactions within the protomer $(c)$. Final stabilization is attained with the proper folding of $\mathrm{SM}^{\prime}$ and of the $\mathrm{Cl}_{\mathrm{A}}$ and $\mathrm{Cl}_{\mathrm{B}}$ motifs that allow the binding of chloride ions (green spheres) $(d)$. The two protomers in the hexamer are now ready to be joined by sulfilimine bonds (red lines).

$\alpha 2 \mathrm{NC}_{\text {homo }}$ and $\alpha 4 \mathrm{NC1}_{\text {homo }}$ could represent stable physiological assemblies, but the structures of the individual chains in these noncanonical oligomers might provide a frozen glimpse of transient conformational states in the process of $\mathrm{NC1}$ folding and hexamer building. This is supported by the identical folding of many parts of these noncanonical oligomers and canonical structures, particularly in polar layers ( $\beta$-sheets I and $\mathrm{I}^{\prime}$ ), and the restriction of flexible regions in both noncanonical structures to essentially the same sequence stretches belonging to the equatorial layer (mainly $\beta$-sheets II and $\mathrm{II}^{\prime}$ ).

As already mentioned, $\beta$-sheets $\mathrm{I} / \mathrm{I}^{\prime}$ could act as a stable structural core nucleating the $\mathrm{NC1}$ domains, which could prevent their final folding until integrated into a trimer, since the SM2 would have to be inserted into $\beta$-sheet $\mathrm{II}^{\prime}$ of an adjacent subunit. A plausible scenario for $\mathrm{NC1}$ equatorial layer folding that is compatible with our observations with noncanonical oligomers would involve first the formation of the central $\beta$-barrellike structure by SM1/SM1', with the immediate insertion of $\mathrm{SM} 2^{\prime}$ into the forming $\beta$-sheet II of the 
same subunit. This would make SM2 ready for insertion into the $\mathrm{SM}^{\prime}$ of an adjacent folding protomer. Following this insertion, $\beta$-sheet $\mathrm{II}^{\prime}$ would acquire its final structure. The process of a central $\beta$-barrel-like organization reduces the size of the central tunnel, restricting the number of monomers in the protomer to three, which is further stabilized by the presence of the triple-helical collagenous domain. Subsequently, flexible loops $\left(\mathrm{Cl}_{\mathrm{A}}\right.$ and $\mathrm{Cl}_{\mathrm{B}}$ motifs $)$ at the equatorial plane of the protomer could acquire a competent conformation for chloride binding, leading to the final assembly of the hexamer involving the interaction of two protomers through their flat surfaces in a chloride-rich medium, i.e. the extracellular fluid, with further hexamer stabilization by sulfilimine cross-linkings (Fig. 6). In the previous description, the potential participation of an uncharacterized cellular machinery in assisting this process of chain sorting, folding and assembly has not been taken into account. It has recently been reported that the production of an $\alpha 5$ network in cancer cell lines is dependent on the expression and activity of the Goodpasture antigen-binding protein (GPBP). This strongly suggests that this machinery exists and that GPBP forms an important part of it (Revert et al., 2018).

Our present work helps to clarify why missense mutations affecting $\mathrm{NC1}$ domains in Alport's syndrome are more frequently found in the structural core of the $\mathrm{NC1}$ domain. Given the key role of $\beta$-sheets $\mathrm{I} / \mathrm{I}^{\prime}$ in subunit folding that is suggested by our findings, mutations in this region are likely to have increased visibility ('eloquent mutations') since they will have a higher impact on the final structure in comparison to mutations in structurally less relevant parts. Thus, the genetic variants of Alport's syndrome owing to missense mutations in this domain can be considered to cause a folding disease, as is supported by the occurrence of the unfolded protein response and of endoplasmic reticulum stress in Alport's syndrome (Gould et al., 2007; Pieri et al., 2014). With this in mind, chemical chaperones could be used to help to restore the proper conformation and folding in cases with certain missense mutants (Murray et al., 2014; Wang et al., 2017).

In Goodpasture's autoimmune disease, the autoepitopes are immunoreactive only when the native $\alpha 345 \mathrm{NC} 1$ hexamers are dissociated into monomers or dimer subunits (Hudson et al., 2003; Pedchenko et al., 2010). The underlying mechanism has been attributed to conformational changes forming epitopes in dissociated $\alpha 3$ and $\alpha 5$ subunits that bind the pathogenic autoantibodies (Calvete et al., 2006; Pedchenko et al., 2010). Our crystal structures of the $\alpha 3 \mathrm{NC} 1$ and $\alpha 5 \mathrm{NC} 1$ homohexamers provide the first view of the arrangement of the $\mathrm{E}_{\mathrm{A}}$ and $\mathrm{E}_{\mathrm{B}}$ regions, which are known critical components of two epitopes that reside in the dissociated $\alpha 3$ and $\alpha 5$ subunits of native $\alpha 345 \mathrm{NC} 1$ hexamers. In the homohexamers these regions are exposed on the surface and are presumably unreactive to antibody binding, as they are in the native $\alpha 345 \mathrm{NC} 1$ hexamers. This knowledge of $\mathrm{E}_{\mathrm{A}}$ and $\mathrm{E}_{\mathrm{B}}$ structures will be critical in elucidating the structural mechanism that enables epitope presentation only in dissociated subunits. Potentially, the mechanism will shed light on the aetiology of Goodpasture's disease.
It is conceivable that the extreme structural stability of the regions visualized here as pertaining to the $E_{A}$ and $E_{B}$ epitopes could be related to a high antigenicity because of the presentation of a single conformer, while other areas of the protein with higher flexibility could present many different conformations, populated with reduced frequencies, to the antibody-making machinery. The preference of Goodpasture antibodies to recognize the $\alpha 3 \mathrm{NC} 1$ chain could be explained by its sequence composition, although the recent description of Goodpasture's disease with autoantibodies reactive exclusively to $\alpha 5 \mathrm{NC1}$ (Cui et al., 2016) opens the door to additional factors being implicated in pathogenesis. Anti- $\alpha 3 \mathrm{NC1}$ autoantibodies are found in most Goodpasture's disease patients. The presence of $\alpha 5 \mathrm{NC1}$-specific autoantibodies could be considered to be to some extent atypical or exceptional in the context of Goodpasture's disease, and thus $\alpha 3 \mathrm{NC1}$ must exhibit some singularity that makes it more susceptible to undergoing autoimmune attack in comparison with other chains.

\section{Acknowledgements}

We thank the IBV-CSIC Crystallogenesis Facility for protein crystallization screening. The experimental results reported in this article are derived from measurements made at the ESRF (Grenoble, France), Diamond Light Source (Didcot, England) and ALBA (Cerdanyola del Valles, Spain) synchrotrons. We thank the staff of the beamlines used for assistance. X-ray diffraction data collection was supported by Diamond Light Source block allocation group (BAG) Proposals MX10121 and MX14739 and Spanish Synchrotron Radiation Facility ALBA Proposals 2015071314 and 2017072262.

\section{Funding information}

The following funding is acknowledged: Spanish Government (Ministerio de Economia y Competitividad; grant Nos. BIO2013-42619 and BIO2016-78571-P to Alberto Marina; grant Nos. SAF 2009-10703, IPT-2011-1527-010000 and RTC2014-2415-1 to Juan Saus; grant No. BFU2016-78606-P to Patricia Casino; grant No. BFU2014-58229-P to Vicente Rubio; contract No. RYC-2014-16490 to Patricia Casino); Valencian Government Prometeo program (grant No. II/2014/ 029 to Alberto Marina, Vicente Rubio and Juan Saus); National Institutes of Health (grant NIH-DK18381 to Billy G. Hudson); European Community's Seventh Framework Programme FP7/2007-2013 (BioStruct-X; grant No. 283570); and a visiting researcher grant from the program 'Atracció de Talent' of the University of Valencia to Billy G. Hudson.

\section{References}

Anazco, C., Lopez-Jimenez, A. J., Rafi, M., Vega-Montoto, L., Zhang, M.-Z., Hudson, B. G. \& Vanacore, R. M. (2016). J. Biol. Chem. 291, 25999.

Bhave, G., Cummings, C. F., Vanacore, R. M., Kumagai-Cresse, C., Ero-Tolliver, I. A., Rafi, M., Kang, J.-S., Pedchenko, V., Fessler, L. I., Fessler, J. H. \& Hudson, B. G. (2012). Nature Chem. Biol. 8, 784-790. 
Borza, D. B., Bondar, O., Ninomiya, Y., Sado, Y., Naito, I., Todd, P. \& Hudson, B. G. (2001). J. Biol. Chem. 276, 28532-28540.

Boutaud, A., Borza, D.-B., Bondar, O., Gunwar, S., Netzer, K.-O., Singh, N., Ninomiya, Y., Sado, Y., Noelken, M. E. \& Hudson, B. G. (2000). J. Biol. Chem. 275, 30716-30724.

Bradford, M. M. (1976). Anal. Biochem. 72, 248-254.

Brown, K. L., Cummings, C. F., Vanacore, R. M. \& Hudson, B. G. (2017). Protein Sci. 26, 2151-2161.

Calvete, J. J., Revert, F., Blanco, M., Cervera, J., Tárrega, C., Sanz, L., Revert-Ros, F., Granero, F., Pérez-Payá, E., Hudson, B. G. \& Saus, J. (2006). Proteomics, 6, S237-S244.

Campbell, K. P. \& Stull, J. T. (2003). J. Biol. Chem. 278, 12599 12600.

Court, F. A., Wrabetz, L. \& Feltri, M. L. (2006). Curr. Opin. Neurobiol. 16, 501-507.

Crockett, D. K., Pont-Kingdon, G., Gedge, F., Sumner, K., Seamons, R. \& Lyon, E. (2010). Hum. Mutat. 31, E1652-E1657.

Cui, Z., Zhao, M.-H., Jia, X.-Y., Wang, M., Hu, S.-Y., Wang, S.-X., Yu, F., Brown, K. L., Hudson, B. G. \& Pedchenko, V. (2016). J. Autoimmun. 70, 1-11.

Cummings, C. F. et al. (2016). J. Cell Biol. 213, 479-494.

Daley, W. P. \& Yamada, K. M. (2013). Curr. Opin. Genet. Dev. 23, 408-414.

Dölz, R., Engel, J. \& Kühn, K. (1988). Eur. J. Biochem. 178, 357-366.

Emsley, P., Lohkamp, B., Scott, W. G. \& Cowtan, K. (2010). Acta Cryst. D66, 486-501.

Fidler, A. L., Darris, C. E., Chetyrkin, S. V., Pedchenko, V. K., Boudko, S. P., Brown, K. L., Jerome, W. G., Hudson, J. K., Rokas, A. \& Hudson, B. G. (2017). Elife, 6, e24176.

Fidler, A. L., Boudko, S. P., Rokas, A. \& Hudson, B. G. (2018). J. Cell Sci. 131, jcs203950.

Gould, D. B., Marchant, J. K., Savinova, O. V., Smith, R. S. \& John, S. W. (2007). Hum. Mol. Genet. 16, 798-807.

Gould, D. B., Phalan, F. C., Breedveld, G. J., van Mil, S. E., Smith, R. S., Schimenti, J. C., Aguglia, U., van der Knaap, M. S., Heutink, P. \& John, S. W. (2005). Science, 308, 1167-1171.

Gozalbo-Rovira, R., Rodríguez-Díaz, J., Saus, J. \& Cervera, J. (2013). Kidney Int. 83, 438-445.

Gunwar, S., Ballester, F., Noelken, M. E., Sado, Y., Ninomiya, Y. \& Hudson, B. G. (1998). J. Biol. Chem. 273, 8767-8775.

Hagios, C., Lochter, A. \& Bissell, M. J. (1998). Philos. Trans. R. Soc. Lond. B Biol. Sci. 353, 857-870.

Hayward, S. \& Berendsen, H. J. (1998). Proteins, 30, 144-154.

Hudson, B. G., Tryggvason, K., Sundaramoorthy, M. \& Neilson, E. G. (2003). N. Engl. J. Med. 348, 2543-2556.

Hynes, R. O. (2009). Science, 326, 1216-1219.

Kabsch, W. (2010). Acta Cryst. D66, 125-132.

Kalluri, R. (2003). Nature Rev. Cancer, 3, 422-433.

Khoshnoodi, J., Cartailler, J.-P., Alvares, K., Veis, A. \& Hudson, B. G. (2006). J. Biol. Chem. 281, 38117-38121.

Khoshnoodi, J., Sigmundsson, K., Cartailler, J.-P., Bondar, O., Sundaramoorthy, M. \& Hudson, B. G. (2006). J. Biol. Chem. 281, 6058-6069.

Konagurthu, A. S., Whisstock, J. C., Stuckey, P. J. \& Lesk, A. M. (2006). Proteins, 64, 559-574.
Kuo, D. S., Labelle-Dumais, C. \& Gould, D. B. (2012). Hum. Mol. Genet. 21, R97-110.

Laemmli, U. K. (1970). Nature (London), 227, 680-685.

McCoy, A. J., Grosse-Kunstleve, R. W., Adams, P. D., Winn, M. D., Storoni, L. C. \& Read, R. J. (2007). J. Appl. Cryst. 40, 658-674.

Murray, L. S., Lu, Y., Taggart, A., Van Regemorter, N., Vilain, C., Abramowicz, M., Kadler, K. E. \& Van Agtmael, T. (2014). Hum. Mol. Genet. 23, 283-292.

Murshudov, G. N., Skubák, P., Lebedev, A. A., Pannu, N. S., Steiner, R. A., Nicholls, R. A., Winn, M. D., Long, F. \& Vagin, A. A. (2011). Acta Cryst. D67, 355-367.

Netzer, K.-O., Leinonen, A., Boutaud, A., Borza, D.-B., Todd, P., Gunwar, S., Langeveld, J. P. M. \& Hudson, B. G. (1999). J. Biol. Chem. 274, 11267-11274.

Papadopoulos, J. S. \& Agarwala, R. (2007). Bioinformatics, 23, $1073-$ 1079.

Pastor-Pareja, J. C. \& Xu, T. (2011). Dev. Cell, 21, 245-256.

Pedchenko, V., Bondar, O., Fogo, A. B., Vanacore, R., Voziyan, P., Kitching, A. R., Wieslander, J., Kashtan, C., Borza, D.-B., Neilson, E. G., Wilson, C. B. \& Hudson, B. G. (2010). N. Engl. J. Med. 363, 343-354.

Pieri, M. et al. (2014). J. Am. Soc. Nephrol. 25, 260-275.

Raya, A., Revert, F., Navarro, S. \& Saus, J. (1999). J. Biol. Chem. 274, 12642-12649.

Revert, F. et al. (2018). Oncotarget, 9, 11020-11045.

Revert, F., Ventura, I., Martínez-Martínez, P., Granero-Moltó, F., Revert-Ros, F., Macías, J. \& Saus, J. (2008). J. Biol. Chem. 283, 30246-30255.

Rhodes, J. M. \& Simons, M. (2007). J. Cell. Mol. Med. 11, 176-205.

Robertson, W. E., Rose, K. L., Hudson, B. G. \& Vanacore, R. M. (2014). J. Biol. Chem. 289, 25601-25610.

Sado, Y., Boutaud, A., Kagawa, M., Naito, I., Ninomiya, Y. \& Hudson, B. G. (1998). Kidney Int. 53, 664-671.

Sanes, J. R. (2003). J. Biol. Chem. 278, 12601-12604.

Sillat, T., Saat, R., Pöllänen, R., Hukkanen, M., Takagi, M. \& Konttinen, Y. T. (2012). J. Cell. Mol. Med. 16, 1485-1495.

Sundaramoorthy, M., Meiyappan, M., Todd, P. \& Hudson, B. G. (2002). J. Biol. Chem. 277, 31142-31153.

Than, M. E., Henrich, S., Huber, R., Ries, A., Mann, K., Kühn, K., Timpl, R., Bourenkov, G. P., Bartunik, H. D. \& Bode, W. (2002). Proc. Natl Acad. Sci. USA, 99, 6607-6612.

Timpl, R., Wiedemann, H., van Delden, V., Furthmayr, H. \& Kühn, K. (1981). Eur. J. Biochem. 120, 203-211.

Vanacore, R., Ham, A. J., Voehler, M., Sanders, C. R., Conrads, T. P., Veenstra, T. D., Sharpless, K. B., Dawson, P. E. \& Hudson, B. G. (2009). Science, 325, 1230-1234.

Vanacore, R. M., Shanmugasundararaj, S., Friedman, D. B., Bondar, O., Hudson, B. G. \& Sundaramoorthy, M. (2004). J. Biol. Chem. 279, 44723-44730.

Wang, D., Mohammad, M., Wang, Y., Tan, R., Murray, L. S., Ricardo, S., Dagher, H., van Agtmael, T. \& Savige, J. (2017). Kidney Int. Rep. 2, 739-748.

Wang, X., Harris, R. E., Bayston, L. J. \& Ashe, H. L. (2008). Nature (London), 455, 72-77.

Winn, M. D. et al. (2011). Acta Cryst. D67, 235-242. 